\title{
Compatible Solute Synthesis and Import by the Moderate Halophile Spiribacter salinus: Physiology and Genomics
}

\author{
María J. León ${ }^{1 \dagger}$, Tamara Hoffmann ${ }^{2 \dagger}$, Cristina Sánchez-Porro ${ }^{1}$, Johann Heider ${ }^{2,3}$, \\ Antonio Ventosa ${ }^{1 *}$ and Erhard Bremer ${ }^{2,3 *}$
}

\begin{abstract}
${ }^{1}$ Department of Microbiology and Parasitology, Faculty of Pharmacy, University of Seville, Seville, Spain, ${ }^{2}$ Laboratory for Microbiology, Department of Biology, Philipps University of Marburg, Marburg, Germany, ${ }^{3}$ LOEWE-Center for Synthetic Microbiology, Philipps University of Marburg, Marburg, Germany
\end{abstract}

OPEN ACCESS

Edited by:

Satoshi Tsuneda,

Waseda University, Japan

Reviewed by:

Aharon Oren

Hebrew University of Jerusalem, Israel

Tommy Harding,

Dalhousie University, Canada

${ }^{*}$ Correspondence:

Antonio Ventosa

ventosa@us.es

Erhard Bremer

bremer@staff.uni-marburg.de

${ }^{\dagger}$ These authors have contributed equally to this work.

Specialty section:

This article was submitted to Microbial Physiology and Metabolism,

a section of the journal

Frontiers in Microbiology

Received: 01 November 2017

Accepted: 17 January 2018

Published: 15 February 2018

Citation:

León MJ, Hoffmann T,

Sánchez-Porro C, Heider $J$, Ventosa A and Bremer E (2018)

Compatible Solute Synthesis and Import by the Moderate Halophile Spiribacter salinus: Physiology and Genomics.

Front. Microbiol. 9:108. doi: 10.3389/fmicb.2018.00108
Members of the genus Spiribacter are found worldwide and are abundant in ecosystems possessing intermediate salinities between seawater and saturated salt concentrations. Spiribacter salinus M19-40 is the type species of this genus and its first cultivated representative. In the habitats of $S$. salinus M19-40, high salinity is a key determinant for growth and we therefore focused on the cellular adjustment strategy to this persistent environmental challenge. We coupled these experimental studies to the in silico mining of the genome sequence of this moderate halophile with respect to systems allowing this bacterium to control its potassium and sodium pools, and its ability to import and synthesize compatible solutes. S. salinus M19-40 produces enhanced levels of the compatible solute ectoine, both under optimal and growth-challenging salt concentrations, but the genes encoding the corresponding biosynthetic enzymes are not organized in a canonical ectABC operon. Instead, they are scrambled (ectAC; ectB) and are physically separated from each other on the $S$. salinus M19-40 genome. Genomes of many phylogenetically related bacteria also exhibit a non-canonical organization of the ect genes. S. salinus M19-40 also synthesizes trehalose, but this compatible solute seems to make only a minor contribution to the cytoplasmic solute pool under osmotic stress conditions. However, its cellular levels increase substantially in stationary phase cells grown under optimal salt concentrations. In silico genome mining revealed that $S$. salinus M19-40 possesses different types of uptake systems for compatible solutes. Among the set of compatible solutes tested in an osmostress protection growth assay, glycine betaine and arsenobetaine were the most effective. Transport studies with radiolabeled glycine betaine showed that S. salinus M19-40 increases the pool size of this osmolyte in a fashion that is sensitively tied to the prevalent salinity of the growth medium. It was amassed in salt-stressed cells in unmodified form and suppressed the synthesis of ectoine. In conclusion, the data presented here allow us to derive a genome-scale picture of the cellular adjustment strategy of a species that represents an environmentally abundant group of ecophysiologically important halophilic microorganisms.

Keywords: Spiribacter, halophiles, hypersaline environments, osmoadaptation, ectoine, trehalose, glycine betaine, arsenobetaine 


\section{INTRODUCTION}

The earth possesses widely distributed hypersaline ecosystems in which the main life-limiting factor is their high salt concentration (Ventosa et al., 1998; Grant, 2004). Most microbiological studies of these extreme ecosystems have been carried out on aquatic habitats, such as saline lakes and marine salterns. Salterns constitute excellent models for the study of the diversity and ecology of microorganisms that either strive or struggle under high-saline growth conditions (Ventosa et al., 2014, 2015).

Bacteria belonging to the genera Halomonas, Chromohalobacter, or Marinobacter can easily be isolated from such hypersaline environments and can readily be obtained in pure culture. Despite this, many of these isolates are now recognized as minor inhabitants of these ecosystems (Ghai et al., 2011; Fernandez et al., 2014a). In contrast, the isolation of the most abundant types of microorganisms from hypersaline habitats is challenging (Ventosa et al., 2014), because their cultivation for laboratory studies is difficult and tedious. As a consequence, the physiology of these microorganisms is typically assessed indirectly through metagenomic approaches (Ventosa et al., 2015), or, at best, studied in a few isolated species serving as proxy (Grote et al., 2012; Swan et al., 2013; Carini et al., 2014; Suh et al., 2015).

Members of the genus Spiribacter are abundant but elusive inhabitants of hypersaline ecosystems. The currently known species of the genus Spiribacter, Spiribacter salinus M19-40 (León et al., 2014), Spiribacter curvatus UAH-SP71 (León et al., 2015), Spiribacter roseus SSL50 (León et al., 2016), and Spiribacter aquaticus SP30 (León et al., 2017), have all been described as moderate halophiles. Depending on the particular species of these Gram-negative bacteria, members of the genus Spiribacter can grow in laboratory media with salinities ranging between 7.5 and $25 \%(\mathrm{w} / \mathrm{v})$ and are worldwide distributed, as inferred from metagenomic studies of saltern ponds from Santa Pola (on the East coast of Spain), Isla Cristina (Southwest of Spain), and San Diego (CA, United States) (Lopez-Perez et al., 2013; Fernandez et al., 2014b; León et al., 2014, 2015; Ventosa et al., 2014). The complete genome sequence of the founding member of the genus Spiribacter, S. salinus M19-40, has recently been reported (León et al., 2013; Lopez-Perez et al., 2013). It is small in size (about $1.74 \mathrm{Mb}$ ) and provides the blueprint for a rather simple metabolic set-up (León et al., 2013; Lopez-Perez et al., 2013).

When one considers the lifestyle of halotolerant or halophilic microorganisms (Ventosa et al., 1998; Oren, 2013), it is important to recall that bacterial cells cannot actively pump water across their cytoplasmic membrane in a directed fashion. As a consequence, microorganisms have to compensate indirectly for water influxes or effluxes instigated by fluctuations in the external salinity in order to balance the magnitude of the vital osmotic gradient across their cytoplasmic membrane, and hence preserve vital turgor (Csonka, 1989; Kempf and Bremer, 1998; Bremer and Krämer, 2000; Roesser and Müller, 2001; Wood, 2011). They accomplish this by accumulating ions and organic osmolytes to raise the osmotic potential of the cytoplasm under hyperosmotic conditions to avoid dehydration and collapse of turgor (Kempf and Bremer, 1998), and they rapidly jettison these compounds through the transient opening of mechanosensitive channels when they are suddenly exposed to hypoosmotic circumstances to avoid an undue rise in turgor and eventual cell rupture (Booth, 2014).

The strategies used by microorganisms for their cellular adjustment to high salinity environments can be classified according to the salt in and salt out schemes (Galinski and Trüper, 1994; Kempf and Bremer, 1998; Wood et al., 2001), physiological osmostress responses that are not necessarily mutually exclusive (Deole et al., 2013; Oren, 2013; Youssef et al., 2014). Microorganisms that use the salt in strategy persistently accumulate large concentrations of $\mathrm{K}^{+}$and $\mathrm{Cl}^{-}$ions via transport, an energetically favorable process (Oren, 2011). However, the resulting high ionic strength of the cytoplasm required, on an evolutionary time scale, compensatory changes in practically the entire proteome in order to keep proteins soluble and to ensure the functionality of key cellular activities (Oren, 2013; Saum et al., 2013; Talon et al., 2014; Warden et al., 2015). This has left an acid signature on the proteome of those microorganisms that employ the salt in strategy and typically confine these bacteria and archaea to high salt habitats where the external salinity does not fluctuate frequently.

Although energetically more expensive (Oren, 2011), the salt out strategy is a physiological flexible osmostress adjustment response as it allows microorganisms to grow over a wide range of external salinities. In its core, it comprises the amassing of a selected class of organic osmolytes, the socalled compatible solutes, and the avoidance of a long-lasting large-scale accumulation of ions (Galinski and Trüper, 1994; da Costa et al., 1998; Kempf and Bremer, 1998; Bremer and Krämer, 2000; Roesser and Müller, 2001; Wood et al., 2001). The physico-chemical attributes of compatible solutes and their high water-solubility make them compliant with the proper folding of proteins and the biochemical functioning of key cellular processes (Bolen and Baskakov, 2001; Ignatova and Gierasch, 2006; Street et al., 2006; Zaccai et al., 2016; Stadmiller et al., 2017). Hence, there was no need to evolutionarily adjust the composition of the proteome to a high ionic strength cytoplasm through the acidification of protein surfaces. Compatible solutes can be accumulated to exceedingly high intracellular levels, and the external salinity dictates their cytoplasmic pool size (Kuhlmann A.U. et al., 2008; Brill et al., 2011; Hoffmann et al., 2013). Important representatives of compatible solutes used by both marine and terrestrial members of the bacteria are trehalose, proline, glycine betaine, ectoine/5-hydroxyectoine, glucosylglycerol, and dimethylsulfoniopropionate (DMSP) (Csonka, 1989; da Costa et al., 1998; Kempf and Bremer, 1998; Roesser and Müller, 2001; Klähn and Hagemann, 2011).

Once accumulated by microorganisms under high salinity growth conditions as stress protectants, compatible solutes are discharged into the environment upon osmotic downshifts through the transient opening of mechanosensitive channels or through cell lysis (Welsh, 2000; Booth, 2014). The released organic osmolytes are valuable environmental resources in a given ecosystem since they can be scavenged by other 
microorganisms via high-affinity transport systems either for their re-use as osmostress protectants (Kempf and Bremer, 1998; Bremer and Krämer, 2000; Wood et al., 2001) or for catabolic purposes (Welsh, 2000; Schwibbert et al., 2011; Schulz et al., 2017a,b).

Members of the genus Spiribacter belong to the family Ectothiorhodospiraceae within the Gammaproteobacteria. They represent the first ecologically defined moderate halophiles and are environmentally abundant inhabitants in various hypersaline ecosystems (Lopez-Perez et al., 2013; Fernandez et al., 2014b; León et al., 2014, 2015, 2016, 2017). Therefore, the type species of this genus, S. salinus M19-40 (León et al., 2014), can be considered as an interesting model organism for studies aiming at understanding how moderate halophiles cope with increased salinity, a trait critical for growth in and lasting colonization of the ecosystem they populate. Here, we have combined physiological approaches and in silico genome mining to derive a comprehensive picture of the salt-stress adjustment systems that allows $S$. salinus M19-40 to be an ecophysiological successful bacterium in hypersaline environments.

\section{MATERIALS AND METHODS}

\section{Chemicals}

Glycine betaine, $\gamma$-butyrobetaine, and carnitine were purchased from Sigma-Aldrich (Steinheim, Germany). Arsenobetaine was obtained from Argus Chemical (Verino, Italy). Crotonobetaine was generously provided by J. Brass (Lonza AG, Visp, Switzerland). DMSP was from a laboratory stock and was custom synthesized as described (Nau-Wagner et al., 1999; Broy et al., 2015). Homobetaine and choline-O-sulfate were kind gifts from G. Nau-Wagner (University of Marburg, Germany), and ectoine and 5-hydroxyectoine were generously provided by bitop AG (Witten, Germany). Radiolabeled $\left[1-{ }^{14} \mathrm{C}\right]$ glycine betaine (55 $\mathrm{mCi} \mathrm{mmol}^{-1}$ ) was purchased from American Radiolabeled Chemicals (St. Louis, MO, United States).

\section{Bacterial Strains, Growth Media, and Culture Conditions}

All experiments were conducted with the type species of the genus Spiribacter, S. salinus strain M19-40 (León et al., 2014). It is available from several culture collections under accession numbers CECT $8282^{\mathrm{T}}$ (Spanish Type Culture Collection), IBRCM $10768^{\mathrm{T}}$ (Iranian Biological Resource Center), and LMG $27464^{\mathrm{T}}$ (Belgian Coordinated Collection of Microorganisms).

Cultures of S. salinus M19-40 (40-ml medium in a $100-\mathrm{ml}$ Erlenmeyer flask) were inoculated from exponentially growing pre-cultures and incubated at $37^{\circ} \mathrm{C}$ in a water bath or aerial shaker set to $180 \mathrm{rpm}$. Their growth was monitored photometrically as optical density at $600 \mathrm{~nm}\left(\mathrm{OD}_{600}\right)$. S. salinus M19-40 was routinely cultivated in a modified SMM medium (León et al., 2014) which has the following composition (w/v): $1.95 \% \mathrm{MgCl}_{2} .6 \mathrm{H}_{2} \mathrm{O}, 3.05 \% \mathrm{MgSO}_{4} .7 \mathrm{H}_{2} \mathrm{O}, 0.05 \% \mathrm{CaCl}_{2}, 0.3 \%$ $\mathrm{KCl}, 0.01 \% \mathrm{NaHCO}_{3}, 0.035 \% \mathrm{NaBr}, 0.5 \%$ casein digest, and $0.11 \%$ sodium pyruvate. The $\mathrm{pH}$ of the growth medium was adjusted to 7.5 with $4 \mathrm{M} \mathrm{KOH}$. The osmolarity of the growth medium was adjusted by adding $\mathrm{NaCl}$ from a 5-M stock solution as required by the set-up of the individual experiments. Solutions of the compatible solutes used for osmostress protection assays were sterilized by filtration and added to media from $100 \mathrm{mM}$ stock solutions to a final concentration of $1 \mathrm{mM}$.

\section{Solute Extraction for the Quantification of Ectoine and Trehalose}

Cultures of S. salinus M19-40 were grown at $37^{\circ} \mathrm{C}$ in SMM containing various amounts of $\mathrm{NaCl}$ until they reached the exponential phase (depending on the $\mathrm{NaCl}$ concentration of the medium the cultures had $\mathrm{OD}_{600}$ values that varied between 0.04 and 0.2 ). Twenty milliliter aliquots were withdrawn from the cultures, the cells were harvested by centrifugation and the pellets were solvent extracted in $1 \mathrm{ml}$ ethanol [20\% (v/v)] for $1 \mathrm{~h}$ at room temperature. After centrifugation $(16,000 \times g$ for $30 \mathrm{~min}$ at $4^{\circ} \mathrm{C}$ ), the cell free extracts containing compatible solutes and other soluble material were recovered and dried at $60^{\circ} \mathrm{C}$ for $24 \mathrm{~h}$. The dried compounds were re-suspended in $120 \mu 1 \mathrm{H}_{2} \mathrm{O}$ and the samples were then centrifuged $\left(16,000 \times g\right.$ for $30 \mathrm{~min}$ at $\left.4^{\circ} \mathrm{C}\right)$ to remove water-insoluble particles. The supernatants of these samples were then subsequently used for quantification of ectoine and trehalose.

\section{Quantification of Ectoine}

Ectoine was detected by high-performance liquid chromatography (HPLC) analysis using an Agilent 1260 Infinity LC system (Agilent, Waldbronn, Germany) and a GROM-SIL Amino 1PR column (GROM, Rottenburg-Hailfingen, Germany) essentially as described (Kuhlmann and Bremer, 2002) with the exception that a 1260 Infinity Diode Array Detector (DAD) (Agilent) was employed, instead of the previously used UV/Vis system. The ectoine content of samples was quantified using the OpenLAB software suite (Agilent). The individual values are given as intracellular ectoine concentration $(\mu \mathrm{M})$ in cultures of S. salinus M19-40 that correspond to an $\mathrm{OD}_{600}$ of 1 .

\section{Quantification of Trehalose}

Trehalose was detected via a coupled enzyme reaction assay using the "Trehalose Assay Kit" purchased from Megazyme (Wicklow, Ireland). In this assay, the trehalose concentration of the samples is quantified by the photometrical determination of NADPH at a wavelength of $340 \mathrm{~nm}$ after the trehalase is stoichiometrically converted into gluconate-6-phosphate and NADPH by the activity of the enzymes trehalose, hexokinase, and glucose-6phosphate dehydrogenase. To avoid background reactions of contaminating sugars, an alkaline borohydride reduction of our samples was performed. The alkaline borohydride reduction and the enzymatic reaction were performed as detailed in the manual of the Megazyme trehalose assay kit with the exception that we calculated the trehalose concentrations of the samples using a standard curve derived from the parallel measurement of trehalose standards with concentrations ranging between 0 and $1000 \mu \mathrm{M}$. The individual values are given as intracellular trehalose concentration ( $\mu \mathrm{M})$ in a S. salinus M19-40 culture that corresponds to an $\mathrm{OD}_{600}$ value of 1 . 


\section{Determination of Glycine Betaine Pools}

Intracellular glycine betaine pools were analyzed in S. salinus M19-40 cells that were cultivated in media with various $\mathrm{NaCl}$ concentrations in the presence of $1 \mathrm{mM}$ unlabeled glycine betaine that was spiked with $0.64 \mu \mathrm{M}$ radiolabeled $\left[1-{ }^{14} \mathrm{C}\right]$ glycine betaine. After the cultures reached late exponential growth phase, 3-ml aliquots were filtrated onto membrane filters (Whatman ME25 0.45 $\mu$ M, GE Healthcare, Munich, Germany). The cells on the filters were washed with isotonic growth medium, and the radioactivity of the cells measured by scintillation counting was used to calculate the intracellular glycine betaine concentrations as described previously (Holtmann et al., 2003). The individual values are given as intracellular glycine betaine concentration $(\mu \mathrm{M})$ in cells that correspond to an $\mathrm{OD}_{600}$ value of 1 . To further prove that the cells accumulate glycine betaine, and no metabolic products of it, we separated the intracellular solute pool of the cells by thin-layer chromatography (TLC). One milliliter samples taken from the cultures used to assess the intracellular glycine betaine pools were harvested by centrifugation $(16,000 \times g$ for $30 \mathrm{~min}$ at $4^{\circ} \mathrm{C}$ ). Lysis of the cells, spotting of the intracellular solutes on a TLC plate (Silica Gel G; MachereyNagel, Düren, Germany) and separation by TLC was performed as described previously (Boch et al., 1994). Radiolabeled compounds were visualized using a Storm 840 phosphorimager (GE Healthcare, Munich, Germany), and the strength of the derived signals was quantified with the ImageQuant ${ }^{\mathrm{TM}}$ software package (GE Healthcare, Munich, Germany); these values were normalized to an $\mathrm{OD}_{600}$ of 1 of the S. salinus M19-40 cultures.

\section{Bioinformatic Analysis of the Osmostress Response Systems of S. salinus M19-40}

The amino acid sequence of the EctC protein of S. salinus M19-40 (SPISAL_06145; NCBI genome accession number CP005963), the diagnostic enzyme of the ectoine biosynthetic route (Ono et al., 1999; Widderich et al., 2014, 2016), was used in a BLAST search analysis at the Integrated Microbial Genomes \& Microbiomes (IMG/M) system of the Department of Energy DOI ${ }^{1}$ (Nordberg et al., 2013). The genomes of the 35 microorganisms exhibiting the most closely related EctC amino acid sequences to the S. salinus M19-40 EctC protein were further analyzed using the toolbox provided by the web-server of the IMG/M database. A phylogenetic tree was built using the IMG/M "distance tree" tool which calculates phylogenetic distances and creates a tree using the alignment of 16S rRNA gene sequences based on the SILVA database ${ }^{2}$ and dnadist and neighbor tools from the PHYLIP package ${ }^{3}$. For cases where the exact gene sequence cannot be found in the SILVA database, the sequence with the highest similarity is automatically selected. Subsequently, the ectC gene neighborhoods of the studied 35 microorganisms were analyzed to assess the occurrence of further ectoine related genes. This assessment was extended

\footnotetext{
${ }^{1}$ http://jgi.doe.gov/data-and-tools/img/

${ }^{2} \mathrm{https} / / /$ www.arb-silva.de/

${ }^{3} \mathrm{http}: / /$ evolution.genetics.washington.edu/phylip/doc/
}

via subsequent BLAST searches using the protein sequences of the ectoine hydroxylase (EctD) (accession number CBV43892.1) and the EctB enzyme (accession number OBX36375) from Halomonas elongata (Schwibbert et al., 2011) as search queries. This identified corresponding EctD and EctB homologs from S. salinus M19-40 and from those $35 \mathrm{~S}$. salinus-related species that contained EctC. The amino acid sequences of the putative ectoine hydroxylases were compared and analyzed for the presence of the EctD consensus sequence (Reuter et al., 2010; Widderich et al., 2014) and residues involved in substrate binding and catalysis of this enzyme (Höppner et al., 2014). The genome sequence of S. salinus M19-40 (León et al., 2013; Lopez-Perez et al., 2013) was also searched for various types of compatible solute synthesis, degradation, and import proteins using query sequences derived from various microorganisms that have been physiologically and genetically studied for these traits in the past. A summary of the templates used for this in silico mining of the S. salinus M19-40 genome and the corresponding E-values of the retrieved S. salinus M19-40 proteins are given in Supplementary Table 1.

\section{Generation of Protein Structure Models}

Modeling of putative glycine betaine ligand-binding proteins from S. salinus M19-40 was conducted using the Swiss-Model protein structure prediction platform ${ }^{4}$ (Arnold et al., 2006). The resulting in silico structure models were compared with those of the crystal structures of corresponding extracellular solute receptor proteins from the Escherichia coli ProU and Bacillus subtilis OpuA ABC-transporters [Protein Database (PDB) accession codes for the ProX::glycine betaine and the OpuAC::glycine betaine complexes are 1R9L and $2 \mathrm{~B} 4 \mathrm{~L}$, respectively) (Schiefner et al., 2004; Horn et al., 2006). An in silico model of a predicted ectoine/5-hydroxyectoine ligand-binding protein (TeaA) of the tripartite ATP-independent periplasmic (TRAP) family transporter TeaABC (Grammann et al., 2002) from S. salinus M19-40 was compared with the crystal structure of the corresponding solute receptor protein from $\mathrm{H}$. elongata (PDB accession code 2VPN) (Kuhlmann S.I. et al., 2008). Graphic representations of the various in silico generated models of glycine betaine and ectoine ligand-binding protein complexes from S. salinus M19-40 were prepared using the PyMOL software package $^{5}$ (Delano, 2002).

\section{RESULTS}

\section{Growth Properties of S. salinus M19-40 in Response to Increases in Environmental Salinity}

To assess the amount of $\mathrm{NaCl}$ needed for its growth and the ability of S. salinus M19-40 to colonize hypersaline habitats, cells were propagated in SMM with increasing concentrations of $\mathrm{NaCl}$ (from 0 to $2.0 \mathrm{M} \mathrm{NaCl}$ ). No growth was detectable up to a salinity of $0.4 \mathrm{M} \mathrm{NaCl}$ (Figure 1), a property expected for a moderate halophile (León et al., 2014; Ventosa et al., 2014, 2015).

\footnotetext{
${ }^{4} \mathrm{http}: / /$ swissmodel.expasy.org/

${ }^{5} \mathrm{http}: / /$ www.pymol.org
} 


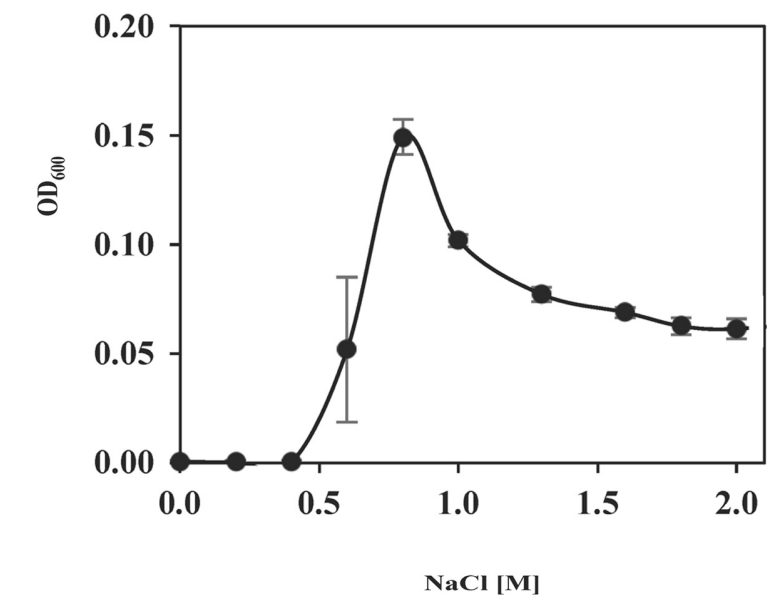

FIGURE 1 | Growth of S. salinus M19-40 at increasing salinities. To determine the growth properties of $S$. salinus M19-40 in response to various concentrations of $\mathrm{NaCl}$ in the medium, the cells were cultured for $90 \mathrm{~h}$ in $\mathrm{SMM}$ at $37^{\circ} \mathrm{C}$ and their growth yields were determined by measuring the $\mathrm{OD}_{600}$. Given are the averaged values and standard deviations derived from three independently grown cultures.

An increase in the salinity up to $0.8 \mathrm{M} \mathrm{NaCl}$ strongly stimulated growth, but further increases (from 1.0 to $2.0 \mathrm{M} \mathrm{NaCl}$ ) successively impaired growth. Hence, S. salinus M19-40 not only depends on a considerable salt concentration for its growth but it can also cope with a broad spectrum of salinities (from 0.6 to $2.0 \mathrm{M} \mathrm{NaCl}$ ). However, its growth is optimally poised for a rather narrow spectrum of salinities (Figure 1).

\section{Mining the Genome Sequence for Systems Mediating $\mathrm{K}^{+}$and $\mathrm{Na}^{+}$ Homeostasis}

The stress response systems of microorganisms which use the salt out adaptation strategy to high-salinity environments typically comprise three interwoven cellular adjustment processes: (i) the import of large quantities of potassium ions, (ii) the accumulation of compatible solutes either through synthesis or uptake, and (iii) the reduction of the ionic strength of the cytoplasm through the export of previously imported potassium ions (Csonka, 1989; Kempf and Bremer, 1998; Bremer and Krämer, 2000; Wood et al., 2001; Wood, 2011). Furthermore, the cell must ensure that the cellular levels of cytotoxic sodium ions are kept at a very low concentration (Gorecki et al., 2014). These processes have, for instance, been intensively studied in E. coli, Salmonella typhimurium, B. subtilis, Corynebacterium glutamicum, H. elongata, and Chromohalobacter salexigens (Csonka, 1989; Kempf and Bremer, 1998; Wood et al., 2001; Morbach and Krämer, 2002; Vargas et al., 2008; Schwibbert et al., 2011; Hoffmann and Bremer, 2016). We took advantage of the analysis of the osmostress response systems of these Gramnegative and Gram-positive microorganisms to derive a plausible in silico picture of the osmostress response system of $S$. salinus M19-40. The results of these efforts are summarized in Figure 2 and are discussed in some detail below.
Fluxes and pools of potassium play key roles in the cellular response to both suddenly imposed and sustained osmotic and salt stress in many microorganisms (Dinnbier et al., 1988; Whatmore et al., 1990). S. salinus M19-40 possesses two copies of Trk-type potassium uptake systems (Figure 2); TrkG (SPISAL_06570 and SPISAL_06575) and TrkH (SPISAL_00315 and SPISAL_00310) (Diskowski et al., 2015). These types of active potassium importers possess a membrane-embedded $\mathrm{K}^{+}$translocating subunit operating in conjunction with a peripheral membrane protein containing an RCK-domain (RCK: regulation of $\mathrm{K}^{+}$channel), which forms a gating ring controlling the activity of the transporter (Diskowski et al., 2015; Szollosi et al., 2016). Genes for other types of well-studied $\mathrm{K}^{+}$import systems, such as Kup, Kdp, and Ktr (Diskowski et al., 2015), or the potassium channel CglK from C. glutamicum (Ochrombel et al., 2011) are not found in the S. salinus M19-40 genome sequence.

Cellular potassium pools are tightly regulated not only through import but also through efflux. We detected a gene (SPISAL_01180) for a KefC-type potassium efflux system (Epstein, 2003) in the genome of S. salinus M19-40. Typically, maximal activity of KefC-type potassium efflux systems is attained through interactions with other types of proteins, such as KefF, KefG, or AmhM. No genes related to these latter three proteins were found in our database search of the S. salinus M1940 genome sequence. We did not find any homologs to other potassium extrusion systems, such as of the B. subtilis $\mathrm{YugO}$ and KhtTU from B. subtilis (Fujisawa et al., 2007; Lundberg et al., 2013).

Further inspection of the $S$. salinus M19-40 genome sequence (León et al., 2013; Lopez-Perez et al., 2013) revealed the presence of genes for a multi-component Mrp sodium extrusion system (Swartz et al., 2005). Mrp-type sodium exporters are widely found in microorganisms and play key roles in monovalent inorganic cation homeostasis, $\mathrm{pH}$ regulation, and energy metabolism (Swartz et al., 2005). The activity of the Mrp system is very important for ensuring growth in high salinity environments; for instance, its mutational inactivation in B. subtilis leads to a pronounced sensitivity to $\mathrm{NaCl}$ (Gorecki et al., 2014). In S. salinus M19-40, the genes for the Mrp-system (SPISAL_06635 to SPISAL_06680) appear to be organized as an operon comprising ten genes (Supplementary Table 1). This is a somewhat unusual number of genes since typical Mrp system consists of six to seven subunits (Swartz et al., 2005) but recent genome surveys of many bacteria and archaea revealed $m r p$ operons with diverse numbers of genes (Ito et al., 2017). An overview of the Mrp proteins from S. salinus M19-40, and their predicted function is given in Supplementary Table 1. Genome searches for the presence of the genes for other types of dedicated sodium extrusion systems such as NatAB, NhaC, and NhaK did not retrieve any hits in S. salinus M19-40.

\section{Mining the Genome Sequence for Compatible Solute Import Systems}

Import of compatible solutes contributes greatly to the cellular defense of many microorganisms to the physiological challenges imposed by high salinity surroundings (Csonka, 1989; Kempf and Bremer, 1998; Bremer and Krämer, 2000; 


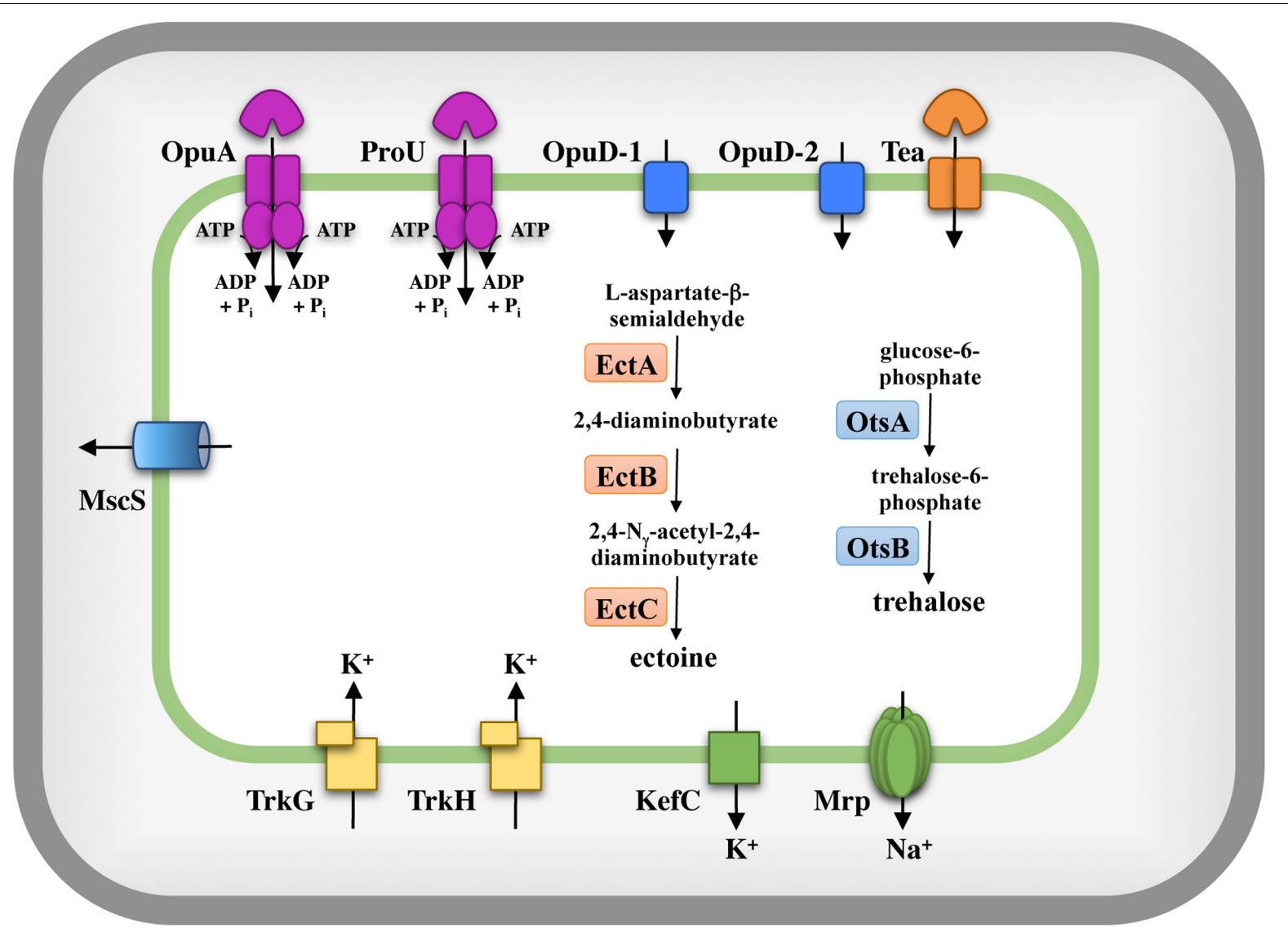

FIGURE 2 | In silico model of the osmostress response systems of S. salinus M19-40. In silico analysis of the genome sequence of S. salinus M19-40 (León et al., 2013; Lopez-Perez et al., 2013) with respect to uptake- and release systems for ions and compatible solutes, compatible solute biosynthesis routes, and mechanosensitive channels, systems which are known from previous studies to be involved in osmostress adaptation in members of the bacteria. The depicted systems are based on bioinformatics data summarized in Supplementary Table 1.

Roesser and Müller, 2001; Wood et al., 2001). We therefore searched the $S$. salinus M19-40 genome for various types of compatible solute transporters and found five distinct systems that can be related to known osmolyte uptake systems (Figure 2).

Two of these predicted osmolyte importers are singlecomponent systems (OpuD-1 and OpuD-2) (SPISAL_05155 and SPISAL_05630, respectively) and these two proteins possess a high degree $(41 \%)$ of amino acid sequence identity to each other. BLAST searches (Altschul et al., 1990) and amino acid comparison of the most closely related proteins demonstrated that the S. salinus M19-40 OpuD-1 and OpuD-2 proteins belong to the betaine-choline-carnitine transporter (BCCT) family (Kappes et al., 1996; Ziegler et al., 2010). Many members of this widespread transporter family mediate the uptake of various types of osmoprotectants, including proline, choline, glycine betaine, arsenobetaine, DMSP, and ectoine (Ziegler et al., 2010; Hoffmann et al., 2017). When the amino acid sequences of the OpuD-1 and OpuD-2 proteins were separately used for BLAST searches using the UniProt knowledgebase (Pundir et al., 2017), the list of the top 17-20 recovered hits was very similar and included functionally characterized members of the BCCT family (Ziegler et al., 2010), including BetS from Rhizobium meliloti, BetT-1 and BetT-2 from Acinetobacter baylyi, OpuD from B. subtilis, BetL from Listeria monocytogenes, EctP, BetP, and LcoP from C. glutamicum, BetT from E. coli, EctT from Virgibacillus pantothenticus, and several BCCT-system from Vibrio parahaemolyticus (Supplementary Figure 1).

The S. salinus M19-40 genome also encodes two ABC transporters that are in all likelihood involved in compatible solute uptake; the OpuA and ProU systems (SPISAL_06400 to SPISAL_06410, and SPISAL_05285 to SPISAL_05290, respectively) (Figure 2). The ProU transporter has been intensively studied both in E. coli and in S. typhimurium and is a binding-protein-dependent ABC-type uptake system (Lucht and Bremer, 1994). A crystal structure of the corresponding periplasmic ligand binding protein (ProX) of the E. coli ProU system in complex with glycine betaine has been determined (Schiefner et al., 2004). Our amino acid sequence comparison between the E. coli ProX and SPISAL_05285 proteins revealed a $31 \%$ sequence identity. Our modeling studies of the SPISAL_05285 protein that are based on the ProX::glycine betaine complex (Schiefner et al., 2004) showed that those residues coordinating the glycine betaine ligand within the active site are conserved (Supplementary Figure 4). 
The second predicted ABC-type osmostress protectant uptake system of S. salinus M19-40 (SPISAL_06400 to SPISAL_06410) is related to the OpuA glycine betaine transporter from B. subtilis (Kempf and Bremer, 1995). The substrate-binding protein (OpuAC) from the Gram-positive bacterium B. subtilis is a lipoprotein tethered to the outer face of the cytoplasmic membrane, whereas the corresponding protein (SPISAL_06400) from the Gram-negative bacterium S. salinus M19-40 is a predicted periplasmic protein (León et al., 2013, 2014). In comparison with the OpuAC crystal structure (Horn et al., 2006), the two lobes of the SPISAL_06400 protein are inversed, a phenomenon that has previously been observed for a substantial group of OpuAC-related proteins (Smits et al., 2008; Wolters et al., 2010). When this is taken into account and an OpuAC-based homology model of the SPISAL_06400 protein is constructed (Supplementary Figure 5A), we found that the aromatic cage coordinating the positively charged trimethylammonium head-group of glycine betaine within the binding site of the $B$. subtilis OpuAC protein via cation- $\pi$ interactions is strictly conserved in the SPISAL_06400 protein (Supplementary Figure 5B). It is worth noting in this context that the E. coli ProU and the B. subtilis OpuA systems provide the cells with uptake capacity for various types of osmostress protectants (Haardt et al., 1995; Hoffmann and Bremer, 2011).

Finally, we also found genes for a TeaABC-type TRAP transporter (SPISAL_01885 to SPISAL_01895) (Figure 2) in the genome of S. salinus M19-40. The prototype of this transporter was characterized in H. elongata (Grammann et al., 2002) where it functions as an import system for ectoine and 5-hydroxyectoine when they are taken up as osmostress protectants. The TeaABC transporter also serves as a recycling system for newly synthesized ectoines that either leak or are actively exported from osmotically stressed $H$. elongata cells, a function that becomes apparent in a $H$. elongata mutant with a genetically inactivated TeaABC system (Grammann et al., 2002). The activity of many TRAP transporters is energized by sodium gradients, and consequently, these types of binding-protein-dependent import systems are particularly prevalent in microorganisms living either in marine or in highly saline habitats (Mulligan et al., 2011).

The ligand-binding protein (SPISAL_01895) of the TeaABC system of $S$. salinus M19-40 exhibits an amino acid sequence identity of 78 and $57 \%$ to the corresponding proteins (TeaA and UehA) from the halophile $H$. elongata and the marine bacterium Ruegeria pomeroyi DSS-3, respectively (Grammann et al., 2002; Lecher et al., 2009). This latter protein is part of a substrateinducible ectoine/5-hydroxyectoine import system (UehABC) used by $R$. pomeroyi DSS-3 to scavenge these compounds as nutrients from environmental sources (Schulz et al., 2017a,b). Despite that the TeaABC and UehABC transporters are used for different physiological purposes, the crystal structures of the 5-hydroxyectoine ligand-binding proteins TeaA and UehA are closely related to each other and their binding sites are virtually superimposable (Kuhlmann S.I. et al., 2008; Lecher et al., 2009). Modeling of the SPISAL_01895 binding protein based on the $H$. elongata TeaA crystal structure (Kuhlmann S.I. et al., 2008) revealed a putative ligand-binding site in which the residues mediating binding of ectoines are strictly conserved (Supplementary Figure 6). Hence, the TeaABC system of S. salinus M19-40 seems to be an ectoine/5-hydroxyectoinespecific uptake system.

A BLAST search using either the TeaA or UehA proteins as the search query identified the presence of an additional TRAP transporter in S. salinus M19-40 (SPISAL_04635 to SPISAL_04645). However, a closer inspection of the corresponding ligand-binding protein (SPISAL_04635) revealed that key determinants for ectoine/5-hydroxyectoine binding by the solute receptors TeaA (Kuhlmann S.I. et al., 2008) and UehA (Lecher et al., 2009) are missing, suggesting that this particular TRAP transporter is not involved in capturing ectoines from external sources.

Sudden osmotic down-shifts require a very rapid adjustment cellular response since the ensuing influx of water can potentially drive up turgor to values that cannot be restrained by the peptidoglycan sacculus (Booth, 2014). Many microorganisms therefore possess safety valves, the mechanosensitive channels, that open transiently to release ions and organic compounds in order to reduce the osmotic potential of the cytoplasm to curb water influx (Booth, 2014). S. salinus M19-40 possesses a gene (SPISAL_03885) for a mechanosensitive channel of small conductance (MscS) (Figure 2) but it lacks a gene for a channel of large conductance (MscL).

Many microorganisms possess AqpZ-type aquaporins, channels that can mediate accelerated water fluxes (Hedfalk et al., 2006). However, the physiological role of these types of aquaporins for osmotic adjustment of bacteria is not entirely clear (Tanghe et al., 2006) and aqpZ-type genes are frequently not present in many microorganisms (Calamita, 2000). The genome sequence of $S$. salinus strain M19-40 lacks an aqpZ-type gene.

\section{Mining the Genome Sequence for Compatible Solute Synthesis Pathways}

In addition to compatible solute import, the synthesis of these types of physiologically compliant organic osmolytes is a common strategy to adjust the cytoplasmic solute pool under unfavorable growth conditions (Csonka, 1989; da Costa et al., 1998; Kempf and Bremer, 1998; Bremer and Krämer, 2000; Roesser and Müller, 2001; Wood et al., 2001). We found genes for the osmostress-responsive synthesis of trehalose and ectoine in the genome sequence of $S$. salinus M19-40.

Trehalose is a non-reducing disaccharide synthesized widely used as compatible solute by microorganisms. It serves not only as an osmostress protectant, but also confers protection against low- and high-temperature stress and preserves the functionality of macromolecules and cells under desiccation stress (Strom and Kaasen, 1993). Trehalose can be produced in microorganisms through various biosynthetic pathways, the most widely distributed is the route catalyzed by OtsAB enzymes (Avonce et al., 2006). In this biosynthetic pathway, glucose-6-phosphate is used as the precursor and is converted into trehalose-6-phosphate by the $\alpha, \alpha$-trehalose-phosphate synthase (OtsA). This intermediate is then dephosphorylated by the trehalose-6-phosphate phosphatase (OtsB) to form free trehalose (Strom and Kaasen, 1993). S. salinus M19-40 possesses 
the corresponding genes (otsB and otsA; SPISAL_07860 and SPISAL_07870; Figure 2). In E. coli, these genes are transcribed as an osmotically inducible operon (Hengge-Aronis et al., 1991; Strom and Kaasen, 1993), while in S. salinus M19-40 they are separated by a gene (SPISAL_07865) that is predicted to code for a member of the glycoside hydrolase 15 family. This protein family comprises enzymes that can hydrolyze the glycosidic bond between two or more carbohydrates (Bourne and Henrissat, 2001). The precursor for trehalose synthesis, glucose-6-phosphate, is frequently generated through import of glucose via a PTS-transporter (Boos et al., 1990). In our database searches of the $S$. salinus M19-40 genome, we did not find evidence for glucose-, maltose-, or trehalose-specific components (protein EII) for PTS systems for these sugars. However, as already reported for C. salexigens (Vargas et al., 2008), a glucokinase (SPISAL_03590) (Supplementary Table 1) can be found in S. salinus M19-40 that potentially could provide the precursor for trehalose synthesis.

A previous inspection of the S. salinus M19-40 genome sequence indicated that this bacterium should be able to synthesize the compatible solute ectoine (Lopez-Perez et al., 2013). Synthesis of ectoine proceeds from L-aspartate$\beta$-semialdehyde and comprises three enzymatic steps that are catalyzed by L-2,4-diaminobutyrate transaminase (EctB), 2,4diaminobutyrate acetyltransferase (EctA), and ectoine synthase (EctC) to yield the cyclic ectoine molecule [(4S)-2-methyl1,4,5,6-tetrahydropyrimidine-4-carboxylic acid] (Louis and Galinski, 1997; Ono et al., 1999). Some microorganisms are also able to synthesize a hydroxylated derivative of ectoine, the effective cytoprotectant 5-hydroxyectoine, in a stereoand region-specific reaction that is catalyzed by the ectoine hydroxylase (EctD) (Prabhu et al., 2004; Garcia-Estepa et al., 2006; Bursy et al., 2007; Höppner et al., 2014).

The structural genes for the ectoine biosynthetic enzymes are typically organized in an operon (ectABC; Louis and Galinski, 1997; Kuhlmann and Bremer, 2002; Kuhlmann A.U. et al., 2008). The ect $D$ gene can be part of the ect $A B C$ ectoine biosynthetic gene cluster, but it is often found elsewhere in the genome (Widderich et al., 2014, 2016). We found that the ectoine biosynthetic genes present in the genome sequence of $S$. salinus M19-40 are not genetically organized into the canonical ect $A B C$ operon. The ectA and ectC genes (SPISAL_06140 and SPISAL_06145) are found close together and their identification as part of the ectoine biosynthetic route seems straightforward. However, the unambiguous identification of the ect $B$ gene turned out to be a bit more complicated. EctB is a L-2,4-diaminobutyrate2-oxoglutarate transaminase (EC 2.6.1.76) and catalyzes the second step in ectoine biosynthesis (Figure 2; Ono et al., 1999). Transaminases participate in various biological transformations and we used therefore the amino acid sequence of the functionally characterized EctB enzyme from $H$. elongata (Ono et al., 1999; Schwibbert et al., 2011) as a template for a BLAST search of the $S$. salinus M19-40 genome. In this way, we found three EctB-related proteins: SPISAL_02400, SPISAL_06590, and SPISAL_07805 (León et al., 2013; Lopez-Perez et al., 2013). To distinguish the potential EctB enzyme from the two other transaminases, we used the orthology IDs from the KEGG database (Kanehisa et al., 2012). The SPISAL_07805 protein belongs to the orthology group K01845-hemL that comprises enzymes involved in heme biosynthesis. SPISAL_06590 belongs the orthology group K00821-argD comprises enzymes involved in arginine biosynthesis, and indeed the gene encoding SPISAL_06590 is flanked by a gene that is annotated in the S. salinus M19-40 genome sequence (León et al., 2013; LopezPerez et al., 2013) as an ornithine-carbamoyl-transferase (ArgF; SPISAL_06595). Bona fide EctB enzymes belong to the orthology group K00836-ectB but the SPISAL_02400 protein was not listed under this identification number in the KEGG database. Instead, it was affiliated with two orthology groups: K00823-puuE (comprising 4-aminobutyrate aminotransferases) and K00821$\operatorname{argD}$. Since EctB is a L-2,4-diaminobutyrate-2-oxoglutarate transaminase (Ono et al., 1999), it seems to us that SPISAL_02400 participates in ectoine biosynthesis; however, we stress that this functional assignment requires further experimental scrutiny. We note that none of the S. salinus M19-40 SPISAL_02400, SPISAL_06590, and SPISAL_07805 proteins exhibits a high degree of amino acid sequence similarity to EctB proteins encoded by other ectoine biosynthetic gene clusters present in taxonomically relates species (Figure 3; see below).

Ectoine and 5-hydroxyectoine are not only effective osmostress protectants for many microorganisms, but they can also serve as nutrients. Genetic and biochemical analysis of Sinorhizobium meliloti, $R$. pomeroyi DSS-3, and H. elongata have provided insights into the 5-hydroxyectoine and ectoine catabolic route (Jebbar et al., 2005; Schwibbert et al., 2011; Schulz et al., 2017a,b). Only a minority of 5-hydroxyectoine/ectoine producers is also capable of 5-hydroxyectoine/ectoine catabolism (Schulz et al., 2017b). Inspection of the S. salinus M19-40 genome sequence showed that the core set of genes required for the use of ectoines as nutrients is missing.

\section{Non-canonical Genetic Organization of Ectoine Biosynthetic Genes in S. salinus M19-40 and Phylogenetically Related Species}

Since the inspection of the $S$. salinus M19-40 genome sequence revealed an unusual genetic organization of the ectoine biosynthetic genes (Figure 3), we wondered whether this was an isolated incident or if this would occur more frequently. We therefore used the amino acid sequence of the S. salinus M19-40 ectoine synthase (EctC) in an initial BLAST search (Altschul et al., 1990) and analyzed the genetic organization of ectoine and 5-hydroxyectoine biosynthetic genes in the best fitting bacterial strains with known 16S rRNA sequences, resulting in 33 members of the Gammaproteobacteria and three of the Deltaproteobacteria with $>78 \%$ EctC sequence identity. Since EctC is typically used as the diagnostic enzyme for the ectoine biosynthetic route (Louis and Galinski, 1997; Ono et al., 1999), it is conveniently employed in database searches to assess the presence of ectoine biosynthetic genes (Widderich et al., 2014, 2016). Strikingly, only nine of the 35 inspected genomes contained the canonical ect $A B C$ gene cluster (Figure 3 ). In most other cases, the genetic organization for the ectoine 


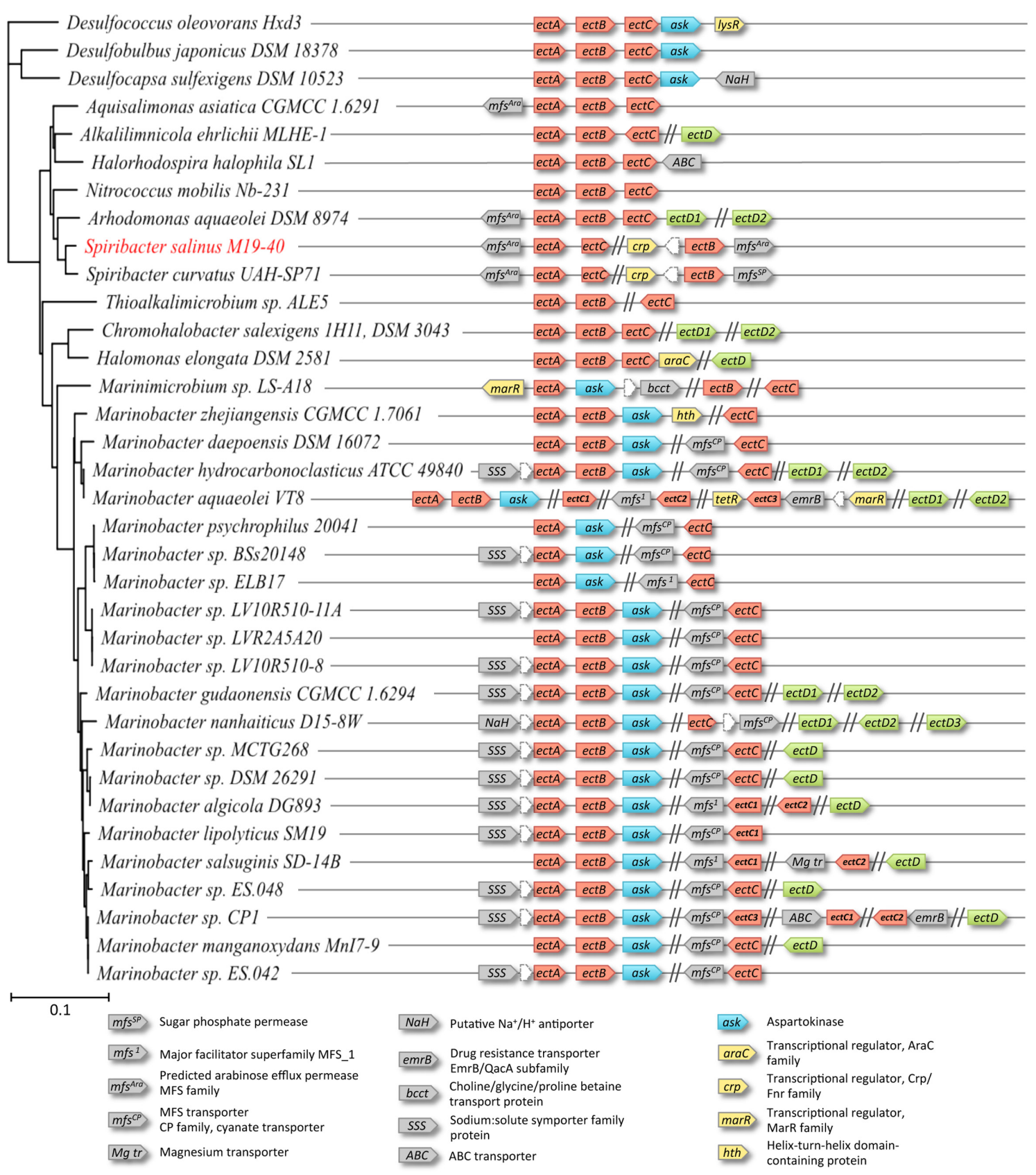

FIGURE 3 | Genetic organization of the ectoine biosynthetic clusters in S. salinus M19-40 and phylogenetically related microorganisms. Genes whose gene products might be involved in ectoine (red), or hydroxyectoine (green) biosynthesis were searched by BLAST analysis in the genomes of S. salinus M19-40 and taxonomically related organisms. The 16S-rRNA based phylogenetic relation of the organisms and the genetic organization of the ect-genes and their genomic neighborhoods are shown. The annotation and distribution of an aspartokinase (ask) associated with ectoine synthesis (blue), putative transporter (gray), or regulator proteins (yellow) encoded in the neighborhood of the ect genes are highlighted.

biosynthetic genes was scrambled. While the co-occurrence of the ectA-ectC genes in an apparent operon was shared by S. salinus M19-40 and S. curvatus UAH-SP71 (Figure 3), the only genome-sequenced Spiribacter species, other members of the family Ectothiorhodospiraceae contain a standard ect $A B C$ cluster with (Arhodomonas) or without (Halorhodospira, Aquisalimonas, Nitrococcus) an ectD gene, or a cluster with divergently oriented
ectC and ectAB genes (Alkalilimnicola). Members of other gammaproteobacterial families contain standard ect gene clusters (Halomonadaceae), clusters with co-localized ectA and $e c t B$ genes, while ectC was located elsewhere in the genome sequences (most Marinobacter species/Alteromonadaceae or Thioalkalimicrobium/Piscirickettsiaceae), or do not show clustering of any ect genes (some Marinobacter strains/ 
Alteromonadaceae or Marinimicrobium/Cellvibrionaceae). Moreover, some of the Marinobacter strains even contain two or three separate copies of ectC genes.

In our dataset, three members of the Marinobacter genus (Marinobacter aquaeolei VT8, Marinobacter salsuginis SD-14B, Marinobacter sp. CP1) possess two to three copies of the ectC gene (Figure 3). The amino acid sequences of the encoded proteins are closely related (Supplementary Figure 2A). Based on an alignment of these sequences we built a phylogenetic tree which suggest that two ectC copies within a given strain evolved from an ancestral gene duplication event and that the third ectC copy present in M. aquaeolei VT8 and Marinobacter sp. CP1 were derived from a more recent gene duplication (Supplementary Figure 2). Interestingly, in the vicinity of these latter additional ect $C$ copies genes are found that encode a transposase.

Many of the studied gammaproteobacterial genome sequences also contained the gene (ectD) encoding the ectoine hydroxylase (Bursy et al., 2007; Höppner et al., 2014), but ectD was, with a single exception, never part of an ect gene cluster (Figure 3). Notably, both S. salinus M19-40 and S. curvatus UAH-SP71 lacked the ectD gene (Figure 3) while only two other members of the Ectothiorhodospiraceae present in our dataset contained ectD genes. The strains affiliated to the Halomonadaceae or Alteromonadaceae mostly contain ectD genes, and a considerable number of them contained even 2, or even 3, ectD copies (Figure 3). This phenomenon has been first functionally studied in $C$. salexigens by assessing the individual contributions of the two EctD-type proteins (referred to as EctD and EctE, respectively) to the overall 5-hydroxyectoine biosynthetic capacity of this bacterium. EctD contributed most to 5-hydroxyectoine production by $C$. salexigens since the expression of ectE was negligible under the studied growth conditions (Vargas et al., 2008). We compared the amino acid sequence of the ectoine hydroxylase from those five microorganisms that possess multiple ectD genes (Figure 3 ) and found that the amino acid sequence of each of these 11 EctDtype proteins is closely related to each other. Each of these proteins possesses the consensus sequence of bona fide ectoine hydroxylases (Supplementary Figure 3A), a stretch of 17 amino acids that is not only of structural importance for the formation of the cupin barrel (Reuter et al., 2010) but contains five residues involved in binding of the iron catalyst, the 2-oxoglutarate co-factor and the 5-hydroxyectoine reaction product (Höppner et al., 2014). A phylogenetic tree build using the amino acid sequence alignment suggests that each copy of the EctD protein present in a given strain has a different evolutionary history (Supplementary Figure 3B). We can, of course, not tell from our analysis in those microorganisms possessing multiple ectD genes, which copy of the EctD enzymes contributes mostly to 5-hydroxyectoine biosynthesis.

Neither the two sequenced Spiribacter species nor any other member of the Ectothiorhodospiraceae contained a gene for a specialized aspartokinase (Ask_Ect), an enzyme that is involved in producing the precursor for ectoine biosynthesis, beta-aspartate-semialdehyde (Reshetnikov et al., 2006; Stöveken et al., 2011). Nevertheless, this type gene (annotated as ask) is co-localized with ectC in 25 of the 35 studied genome sequences, which comprises all Marinococcus and Marinimicrobium strains (Figure 3). The biochemical properties of the Ask_Ect enzyme and its unusual type of feedback regulation by amino acids have been characterized for the corresponding protein from the ectoine/5-hydroxyectoine producer Pseudomonas stutzeri A1501, a plant-root-associated bacterium (Stöveken et al., 2011). Finally, all three strains of the Deltaproteobacteria present in our dataset (Figure 3) contain a conserved standard ect $A B C$ cluster with an added ask gene. However, they lack ectD, as expected from their physiology as strictly anaerobic sulfate reducers of two unrelated families since EctD is an oxygen-depending enzyme (Bursy et al., 2007; Höppner et al., 2014; Widderich et al., 2014). Most of these strains are of marine origin, either as obligate or facultative aerobic or as strictly anaerobic bacteria, which cover all marine lifestyles from pelagic to sediment habitats and may also occur together in tidal pools or saltern ponds. The presence of highly similar copies of apparent ectABC-ask operons in a few strains of sulfate-reducing Deltaproteobacteria affiliated to two unrelated families, and the lack of these genes in any of the genomesequenced related species may be taken as indication for lateral gene transfer events.

In inspecting the genomic context of the ectoine biosynthetic genes, we noticed in many cases genes whose products are annotated as transporters. They belong to different transporter families and are mostly single-component systems (Figure 3). It remains to be seen, if these putative transporters are involved in the uptake of pre-formed ectoines from environmental sources (Kuhlmann et al., 2011), or if they participate in the recovery of newly synthesized ectoines that have been actively excreted (or leaked) across the cytoplasmic membrane (Grammann et al., 2002).

\section{Finely Tuned Synthesis of Ectoine by S. salinus M19-40 in Response to High Salinity}

While the non-canonic arrangement of ectoine biosynthetic genes in a number of microorganisms has been noted before (Widderich et al., 2014, 2016), to the best of our knowledge, ectoine production has not been analyzed in any of them. To study ectoine synthesis by S. salinus M19-40 in response to salt stress in more detail, we quantified the intracellular ectoine pools of the cells by HPLC analysis. For these experiments, we grew S. salinus M19-40 in SMM medium possessing various salinities, extracted from the soluble solutes from the collected cells, and monitored ectoine production levels by HPLC analysis. These data demonstrate that ectoine biosynthesis in S. salinus M1940 is responsive to the degree of salt stress imposed onto the cells (Figures 4A,B). The ectoine content of the cells increased about twofold from a basal level of $80 \mu \mathrm{M}$ in cultures growing under sub-optimal saline conditions (SMM with $0.6 \mathrm{M} \mathrm{NaCl}$ ) to $170 \mu \mathrm{M}$ in cultures propagated at optimal salinity (SMM with $0.8 \mathrm{M} \mathrm{NaCl}$ ) (Figures 4A,B). Although further increases in the salinity of the medium up to $1.3 \mathrm{M} \mathrm{NaCl}$ notably reduced growth yields, the ectoine pool did not increase concomitantly. Only severely growth restricting salt concentrations $(1.6 \mathrm{M} \mathrm{NaCl}$ or higher) appear to trigger further moderate increases of the 


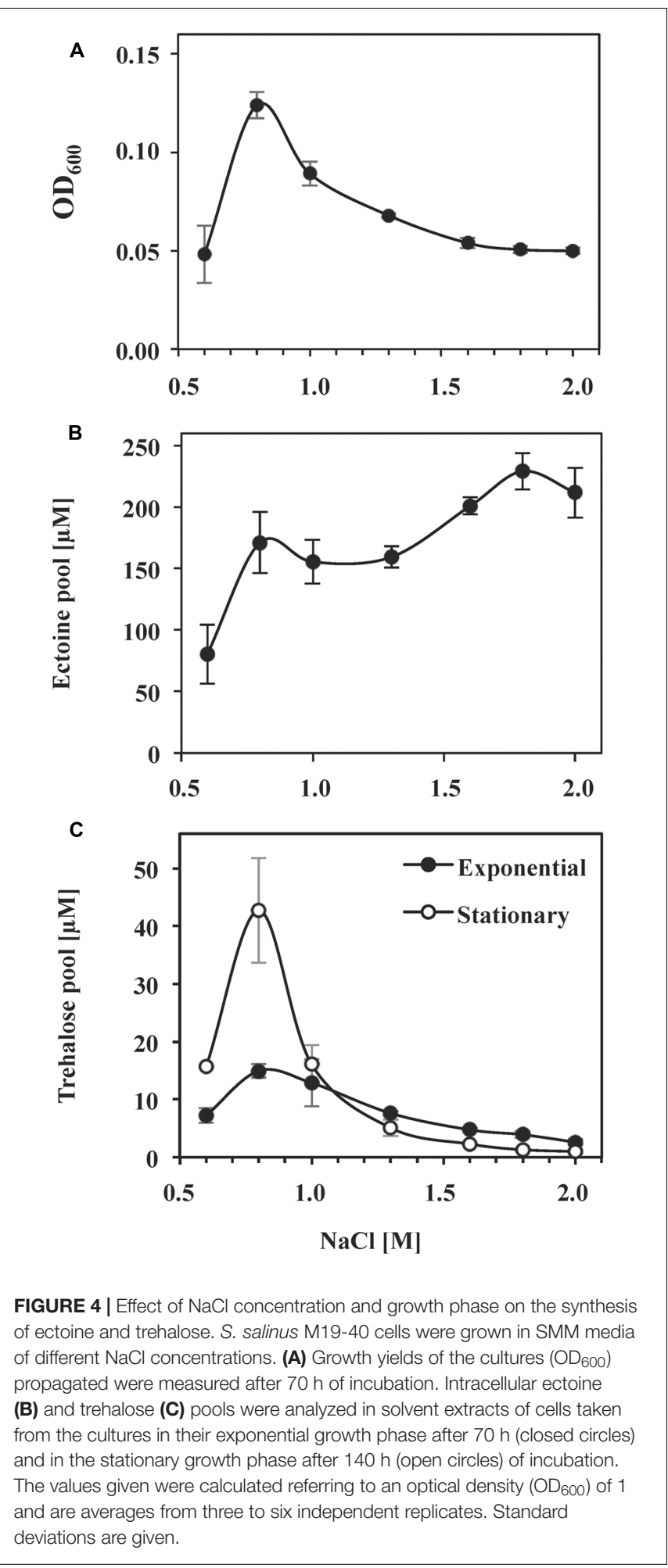

ectoine pools (e.g., $230 \mu \mathrm{M}$ in cultures propagated in SMM with $1.8 \mathrm{M} \mathrm{NaCl}$ ) (Figures 4A,B). Consistent with our finding that the $S$. salinus M19-40 genome sequence does not contain the gene for the ectoine hydroxylase (ectD), we did not detect any 5 -hydroxyectoine in any of the cell extracts.

\section{Synthesis of Trehalose by S. salinus M19-40 in Response to High Salinity and Stationary Phase}

Since mining of the $S$. salinus M19-40 genome sequence indicates that this bacterium possesses the ots $B A$ genes, it should be able to synthesize the compatible solute trehalose (Avonce et al., 2006; Figure 2). We assessed the content of the same cell extracts for the presence of this sugar as used for ectoine quantitation. Trehalose was detected but was present in substantially reduced levels compared to those found for ectoine (Figures 4B,C). This indicates that trehalose contributes only moderately to balance the osmotic gradient across the cytoplasmic membrane of salt-challenged S. salinus M19-40 cells. Trehalose is not only used by microorganisms as an osmostress protectant but also serves as a cytoprotectant against other types of cellular and environmental stresses; e.g., challenges imposed by stationary growth phase (Hengge-Aronis et al., 1991; Strom and Kaasen, 1993). Accordingly, we found a strong increase in trehalose content of stationary phase cells of S. salinus M19-40 propagated under optimal salt concentrations $(0.8 \mathrm{M} \mathrm{NaCl})$, compared to exponentially growing cells (Figure 4C).

\section{Osmostress Protection of S. salinus M19-40 by an Exogenous Supply of Compatible Solutes}

In most microorganisms, relief from high-salinity-imposed stress is accomplished through the import of compatible solutes via various types of high-affinity transport systems (Bremer and Krämer, 2000; Poolman et al., 2004; Krämer, 2010; Wood, 2011). Our in silico analysis of the S. salinus M19-40 genome sequence indicates the presence of several representatives of these systems (Figure 2). To experimentally assess whether S. salinus M1940 is able to take advantage of compatible solutes provided in the growth medium, we tested the stress-protective properties of glycine betaine, arsenobetaine, homobetaine, $\gamma$-butyrobetaine, crotonobetaine, carnitine, choline- $O$-sulfate, and DMSP. For this set of experiments, we grew S. salinus M19-40 in SMM containing 1.6 $\mathrm{M} \mathrm{NaCl}$ in the absence or the presence of $1 \mathrm{mM}$ of the indicated compatible solutes and monitored the growth yield of these cultures. Under such high-salinity conditions (Figure 1), growth of S. salinus M19-40 was severely impaired (Figure 5A). Glycine betaine, and arsenobetaine provided strong osmostress protection while the other tested compatible solutes had more moderate stimulating effects for cell growth (Figure 5A). Since our in silico analysis of the S. salinus M19-40 genome indicates the presence of a TeaABC-related transporter (Grammann et al., 2002; Figure 2) we were particularly surprised that neither externally provided ectoine nor 5-hydroxyectoine afforded any notable osmostress protective effects (Figure 5C).

\section{Osmostress-Responsive Build-up of Glycine Betaine Pools}

The presence of glycine betaine in the medium of high-salinitychallenged S. salinus M19-40 cultures grown in the presence of a broad range of salinities provided substantial osmostress 

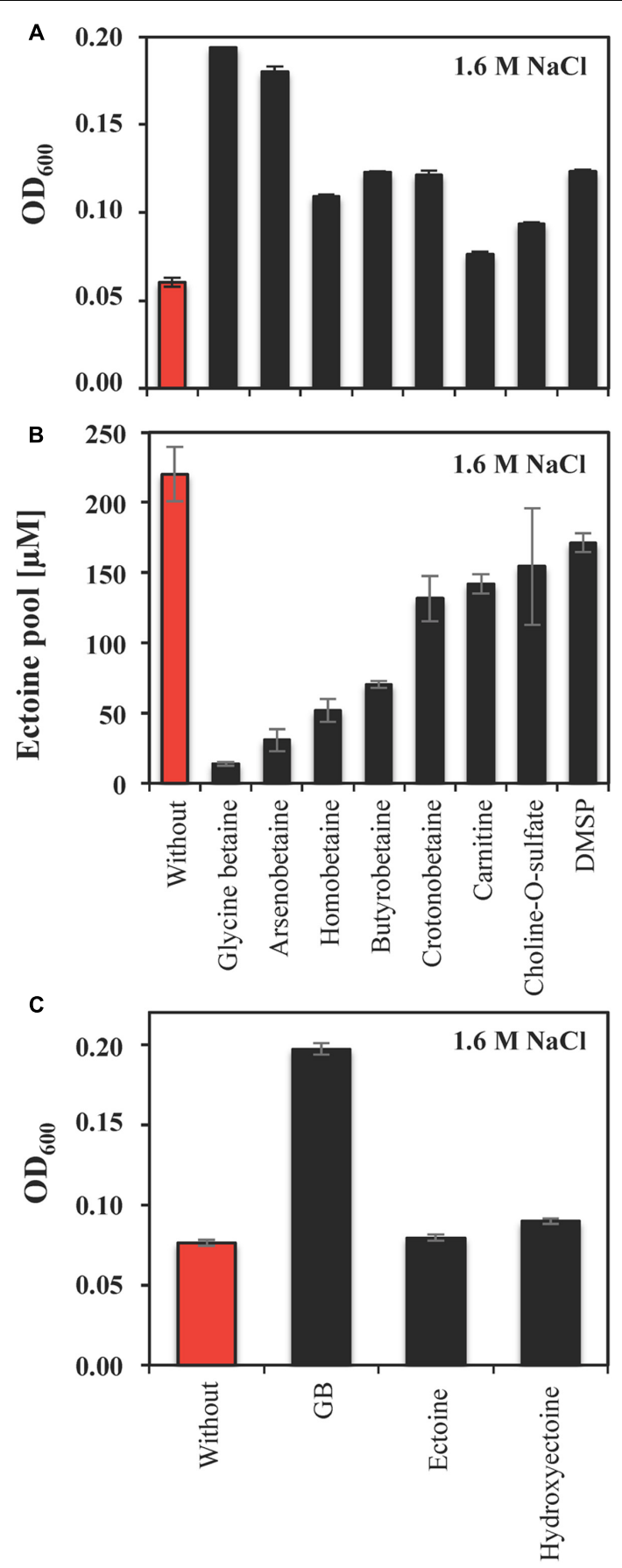

FIGURE 5 | Osmostress protection by compatible solutes and corresponding ectoine pools of $S$. salinus M19-40 cells. (A) Cultures of S. salinus M19-40 were grown at $37^{\circ} \mathrm{C}$ in $\mathrm{SMM}$ containing $1.6 \mathrm{M} \mathrm{NaCl}$. These cultures were propagated either in the absence (red bar) or in the presence of $1 \mathrm{mM}$ of the indicated compatible solutes (black bars), and their growth yields were measured after $70 \mathrm{~h}$. (B) Ethanolic extracts of cells taken from these cultures were analyzed for their ectoine content and were calculated referring to an optical density $\left(\mathrm{OD}_{600}\right)$ of 1 . (C) S. salinus $\mathrm{M} 19-40$ cells were grown at $37^{\circ} \mathrm{C}$ in SMM with $1.6 \mathrm{M} \mathrm{NaCl}$ and in the absence or the presence of $1 \mathrm{mM}$ of glycine betaine, ectoine, or 5-hydroxyectoine, respectively. Growth yields were determined after $70 \mathrm{~h}$. The values given are averages from three independent replicates. Standard deviations are given. protection, even for cells that were cultivated in SMM containing 2.0 $\mathrm{M} \mathrm{NaCl}$ (Figure 6A). We found that radiolabeled glycine betaine was accumulated under steady-state osmotic stress conditions in a linear fashion in response to increases in the external salinity (Figure 6B). As proven by TLC, the amassed glycine betaine was not modified to any other compound by the S. salinus M19-40 cells (Figures 6C,D).

\section{Accumulation of Glycine Betaine Modulates the Ectoine Pools of the Cells}

It is well established that the import and accumulation of osmostress protectants substantially suppresses the synthesis of the endogenously produced compatible solute by the bacterium under study (Hoffmann et al., 2013). This was also the case for ectoine biosynthesis by $S$. salinus M19-40 when the cells were cultivated in the presence of various compatible solutes. The effectiveness of this suppression varied (Figures 5A,B), with glycine betaine and arsenobetaine having the greatest influence on the cellular ectoine pool (Figure 5B). Moreover, the relative osmotic protection and suppression of ectoine biosynthesis appear to deviate for the various tested effectors, suggesting independent effects causing these observations.

Using glycine betaine as an example, we investigated this effect in greater detail in cultures grown in the presence of a broad range of salinities. Glycine betaine provided a substantial degree of osmostress protection when the salinity of the medium exceeded that for optimal growth (Figure 7A). Concomitantly, the intracellular ectoine pool was successively reduced to a basal level, a 17-fold drop when the ectoine pools of S. salinus M19-40 cells grown under optimal salt concentrations $(0.8 \mathrm{M} \mathrm{NaCl})$ were compared with those of severely salt stressed cells (grown in the presence of $2 \mathrm{M} \mathrm{NaCl}$ ) (Figure 7B).

\section{DISCUSSION}

High salinity is a key determinant for the growth of S. salinus M19-40 in the saltern ponds from which it was originally isolated (León et al., 2013, 2014; Lopez-Perez et al., 2013). Such a challenging habitat (Ventosa et al., 2014, 2015) requires active measures by this moderate halophile to counteract the outflow of water from the cells and to optimize the solvent properties of the cytoplasm for biochemical reactions and the functionality of cell components (Bremer and Krämer, 2000; Bolen and Baskakov, 2001; Wood, 2011). Analysis of the in silico-derived proteome and the calculation of isoelectric points of all predicted proteins of $S$. salinus M19-40 suggested that this bacterium uses the salt-out strategy (León et al., 2013; Lopez-Perez et al., 2013). The data presented here support this conclusion. Our experimental and in silico-derived findings highlight the ability of $S$. salinus M19-40 to cope with persistent high-salinity ecosystems through transport-mediated control over its cytoplasmic potassium and sodium pools, the synthesis and accumulation of the effective compatible solute ectoine, and to a minor extent through the synthesis of trehalose. S. salinus M19-40 also can derive a considerable level of osmostress protection through the import of glycine betaine 

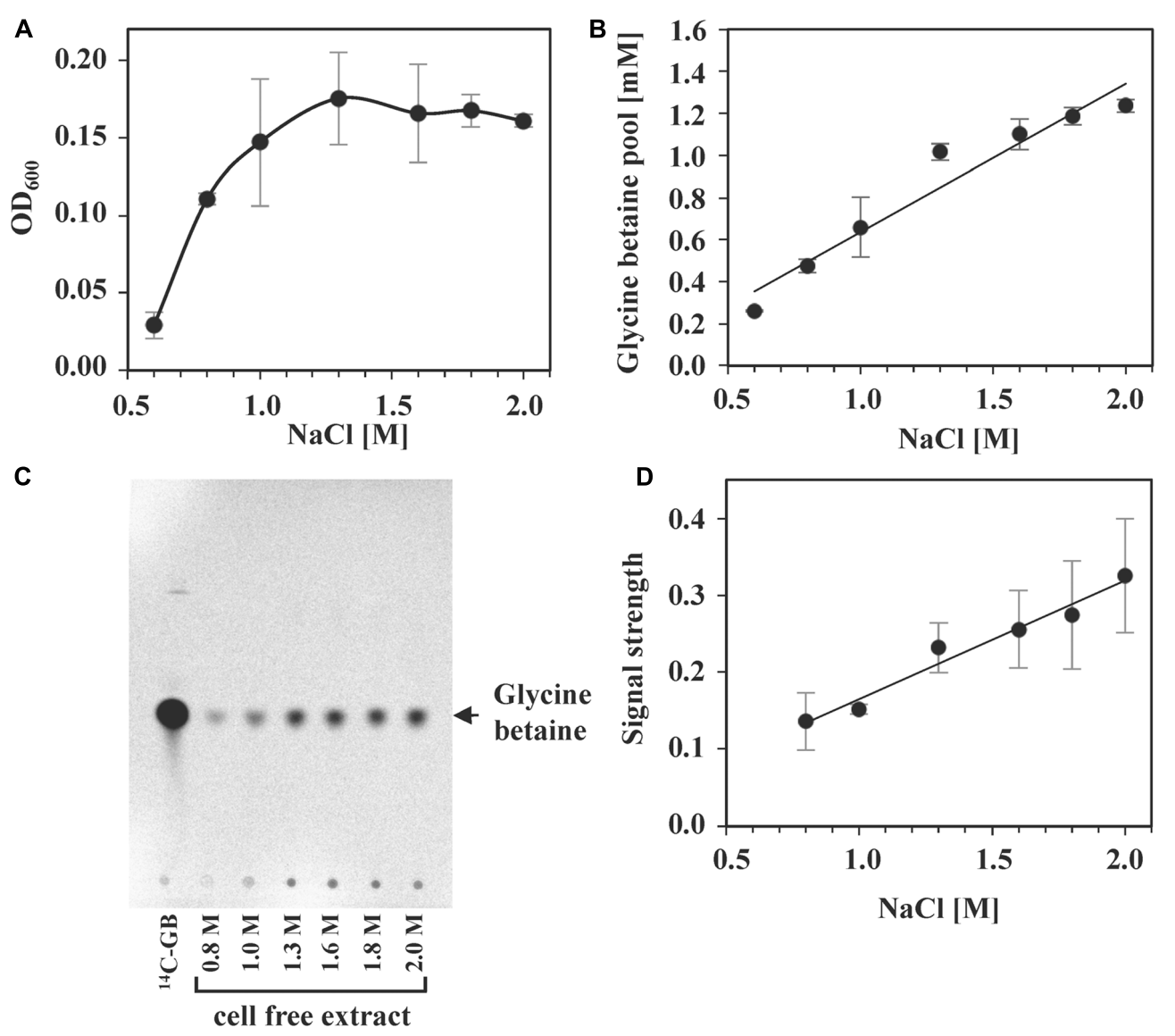

FIGURE 6 | Intracellular glycine betaine accumulation in response to increasing NaCl concentrations. S. salinus M19-40 cultures were grown in three independent replicates, in SMM with increasing $\mathrm{NaCl}$ concentrations in the presence of $1 \mathrm{mM}$ glycine betaine spiked with $0.64 \mu \mathrm{M}\left[1-{ }^{14} \mathrm{C}\right] \mathrm{glycine}$ betaine. (A) Growth yields $\left(\mathrm{OD}_{600}\right)$ of the cultures were measured after $70 \mathrm{~h}$ of incubation at $37^{\circ} \mathrm{C}$. (B) Glycine betaine accumulated by the cells was determined by scintillation counting. The values given for each $\mathrm{NaCl}$ concentration were calculated referring to an optical density $\left(\mathrm{OD}_{600}\right)$ of 1 . (C) Cell-free extracts of the cells were separated by TLC, and radiolabeled compounds were visualized by autoradiography. $\left[1-{ }^{14} \mathrm{C}\right]$ glycine betaine was run on the thin-layer chromatography plate along the samples as a reference. Shown is a representative autoradiograph of one biological replicate. (D) The signals from three independent autoradiographs of TLC plates were quantified, normalized using the $\left[1-{ }^{14} \mathrm{C}\right]$ glycine betaine as internal concentration standard and then calculated referring to the $\mathrm{OD}_{600}$ of the cultures. All values given include averages and standard deviations of three independent replicates.

and its arsenic homolog arsenobetaine. Glycine betaine uptake and trehalose/2-sulfotrehalose biosynthesis are also widely spread mechanisms for osmoadaptation in Halobacteriales, halophilic archaea that often dominate hypersaline habitats (Youssef et al., 2014).

The ability to synthesize the osmostress protectant ectoine is found commonly in nature, including in marine microorganisms, those living in high-salinity ecosystems, and in habitats in which the osmolarity/salinity fluctuates (Pastor et al., 2010; Widderich et al., 2014, 2016). The genetic organization of the ectoine biosynthetic genes from $S$. salinus M19-40 deviate from their canonical arrangement as an ectABC operon (Louis and Galinski, 1997; Kuhlmann A.U. et al., 2008; Pastor et al., 2010; Schwibbert et al., 2011; Widderich et al., 2014, 2016) in that at least one gene is separated from the other two (Figure 3). Our database searches revealed that other non-canonical gene arrangements of the ect biosynthetic genes can also be found in many groups of marine bacteria containing ectC gene products closely related to that of S. salinus M19-40 (Figure 3). To the best of our knowledge, the data reported here address for the first time ectoine biosynthesis in a bacterium with such a scrambled arrangement of ectoine biosynthetic genes. Regardless of the unusual genetic organization of the ectoine biosynthetic genes, S. salinus M19-40 controls the ectoine biosynthetic pathway in a highly coordinated manner and produces ectoine in a fashion that is linked to the level of salinity prevalent in the environment (Figure 4B). Currently, there is no genetic system available for S. salinus M19-40 that would allow the construction of a mutant defective in ectoine biosynthesis to assess the physiological consequence of an ect gene knock-out. However, such a genetic analysis has been carried out in $C$. salexigens, a phylogenetically related ectoine producer (Vargas et al., 2008; Figure 3). As expected, the disruption of the ectoine biosynthetic genes causes osmotic sensitivity (Canovas et al., 1997), and we presume that this would also be the case for $S$. salinus M19-40. 

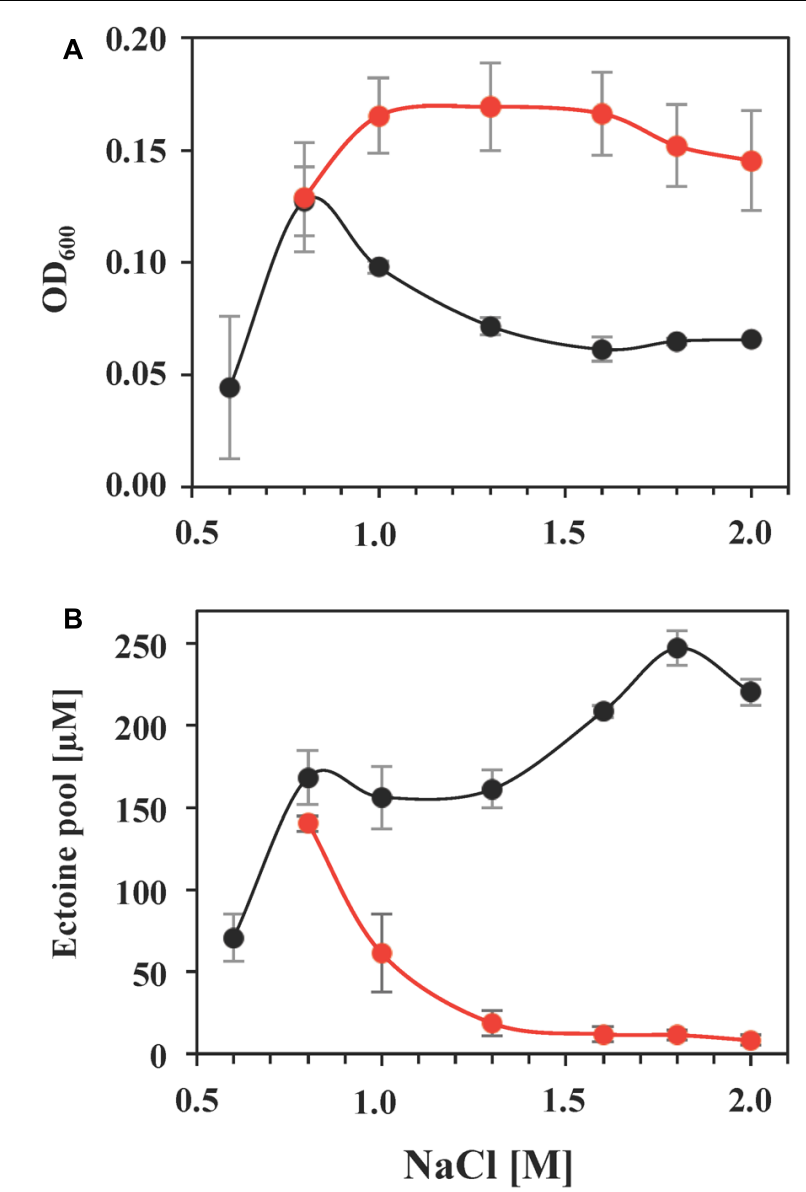

FIGURE 7 | Growth protection by glycine betaine and its influence on the intracellular ectoine pool. S. salinus M19-40 cultures were grown in SMM with increasing $\mathrm{NaCl}$ concentrations either in the absence (black circles) or the presence (red circles) of $1 \mathrm{mM}$ glycine betaine. (A) Growth yields $\left(\mathrm{OD}_{600}\right)$ of the cultures measured after $70 \mathrm{~h}$ of incubation at $37^{\circ} \mathrm{C}$. (B) Ectoine pools were determined in ethanolic extracts of cells harvested from the cultures that were grown to exponential growth phase after $70 \mathrm{~h}$ of incubation. The values given were calculated referring to an optical density $\left(\mathrm{OD}_{600}\right)$ of 1 and are averages from three independent replicates. Standard deviations are given.

Spiribacter salinus M19-40 is an abundant microorganism in saltern ponds with intermediate salinities (León et al., 2013, 2014; Lopez-Perez et al., 2013; Ventosa et al., 2014). As a consequence, cell lysis of ectoine-loaded S. salinus M1940 cells will contribute, in all likelihood, significantly to the environmental ectoine pool that in turn can then be exploited by other inhabitants of this ecosystem, either as an effective osmostress protectant (Pastor et al., 2010) or by using this nitrogen-rich compound as a valuable nutrient (Schwibbert et al., 2011; Schulz et al., 2017a,b). In contrast to H. elongata (Schwibbert et al., 2011) and C. salexigens (Vargas et al., 2006), bacteria that are phylogenetically affiliated to the Halomonadaceae, the genome of S. salinus M19-40 (León et al., 2013; Lopez-Perez et al., 2013) lacks the core genes required for ectoine catabolism (Schwibbert et al., 2011; Schulz et al., 2017a,b). These genes are also not present in the genome sequence of any other bacteria that are phylogenetically related to $S$. salinus M19-40 whose genome sequence we inspected in this study (Figure 3).

The data presented here report for the first time the synthesis of trehalose in any member of the Spiribacter genus (Figure 4C). The low pool size of this compatible solute, however, suggests that it probably plays only a minor role in osmostress adjustment for S. salinus M19-40. There is, however, an up-regulation of the trehalose pool in stationary phase cells suggesting that its production might be primarily associated with the amelioration of types of stresses other than high osmolarity/salinity. For instance, enhanced trehalose production has also been observed when $E$. coli cells enter stationary phase (Giaever et al., 1988; Hengge-Aronis et al., 1991), a growth phase that imposes many constraints onto the bacterial cell (HenggeAronis, 2002).

We found that S. salinus M19-40 can exploit exogenously provided compatible solutes for osmostress protection, thereby broadening the range of salinities where optimal growth can occur (Figures 5A, 7A). Glycine betaine, probably the most widely used compatible solute on Earth (Yancey, 2005), and its arsenic homolog (Popowich et al., 2016; Hoffmann et al., 2017), provided the most effective degree of osmostress protection. Our genome mining data and modeling studies of predicted substratebinding proteins (ProX and OpuAC; Supplementary Figures 4, 5; Schiefner et al., 2004; Horn et al., 2006) of the S. salinus M1940 ProU- and OpuA-type $\mathrm{ABC}$ transporters suggest that these osmolyte importers are involved in scavenging glycine betaine (Figure 2). Arsenobetaine is widely found in marine ecosystems (Popowich et al., 2016) and recent studies with B. subtilis as the model system suggest that in essence any glycine betaine transporter should also be able to import the arsenic homolog (Hoffmann et al., 2017). Indeed, the ABC transporter OpuA and the BCCT-type transporter OpuD from B. subtilis can scavenge arsenobetaine effectively from the growth medium (Hoffmann et al., 2017). The genes for two BCCT-type transporters (OpuD-1; OpuD-2) are found in the genome sequence of S. salinus M19-40 (León et al., 2013; Lopez-Perez et al., 2013). Proteins belonging to the BCCT-family are closely related in their amino acid sequence and topological organization within the membrane, but their substrate specificity cannot be predicted with confidence by simply inspecting their amino acid sequence (Ziegler et al., 2010). The osmotically inducible TRAP-type ectoine/5-hydroxyectoine transporter from $H$. elongata serves for the import of these compatible solutes when they are used as osmostress protectants (Grammann et al., 2002). Amino acid sequence comparison and modeling studies (Supplementary Figure 6) based upon the crystal structure TeaA::ectoine complex of the periplasmic substrate binding protein (Kuhlmann S.I. et al., 2008) strongly suggest that the predicted TeaABC TRAP-transporter from S. salinus M19-40 (Figure 2) should be able to import ectoines. However, contrary to this expectation, exogenously provided ectoines did not serve as osmostress protectants for S. salinus M19-40 (Figure 5C). This is a highly surprising finding and we have currently no reasonable explanation for this observation. In any event, follow-up studies are required to define the precise substrate spectrum of the OpuA, ProU, OpuD-1, OpuD-2, and 
TeaA transporters (Figure 2), and such studies will require the construction of a set of isogenic gene disruption mutations, as, for instance, has been intensively used to define the substrate profile of the osmotically controlled five Opu osmolyte transporters operating in B. subtilis (Hoffmann and Bremer, 2016, 2017).

The import of glycine betaine strongly down-regulated the steady-state ectoine pool of S. salinus M19-40 (Figure 7), indicating that this bacterium prefers glycine betaine as an osmostress protectant. Either because of the superior physicochemical properties of this chemical chaperon (Zaccai et al., 2016; Stadmiller et al., 2017) or to save precursors and biosynthetic resources used for ectoine production (Pastor et al., 2010; Oren, 2011; Widderich et al., 2014). It is known from other studies, that the down-regulation of the ectoine pool in response to the import of glycine betaine or other compatible solutes is primarily the consequence of the down-regulation of the transcription of the ectoine biosynthetic genes (Bestvater et al., 2008; Czech et al., 2018).

In summary, our data suggest that ectoine biosynthesis and glycine betaine/arsenobetaine uptake are key features for the physiological adjustment process of S. salinus M19-40 to sustained but moderately high saline habitats. Nevertheless, the growth profile of S. salinus M19-40 suggests that it is optimally adapted to a rather narrow range of salinities (Figure 1), an observation that is consistent with cultivation experiments and metagenomic data of the habitats from which different members of this genus were isolated (León et al., 2013, 2014, 2015, 2016, 2017; Lopez-Perez et al., 2013). However, its ability to scavenge compatible solutes, in particular glycine betaine and arsenobetaine, from environmental sources will allow S. salinus M19-40 to proliferate under otherwise growthrestricting salinities.

\section{REFERENCES}

Altschul, S. F., Gish, W., Miller, W., Myers, E. W., and Lipman, D. J. (1990). Basic local alignment search tool. J. Mol. Biol. 215, 403-410. doi: 10.1016/S00222836(05)80360-2

Arnold, K., Bordoli, L., Kopp, J., and Schwede, T. (2006). The SWISS-MODEL workspace: a web-based environment for protein structure homology modelling. Bioinformatics 22, 195-201. doi: 10.1093/bioinformatics/ bti770

Avonce, N., Mendoza-Vargas, A., Morett, E., and Iturriaga, G. (2006). Insights on the evolution of trehalose biosynthesis. BMC Evol. Biol. 6:109. doi: 10.1186/ 1471-2148-6-109

Bestvater, T., Louis, P., and Galinski, E. A. (2008). Heterologous ectoine production in Escherichia coli: by-passing the metabolic bottle-neck. Saline Systems 4:12. doi: 10.1186/1746-1448-4-12

Boch, J., Kempf, B., and Bremer, E. (1994). Osmoregulation in Bacillus subtilis: synthesis of the osmoprotectant glycine betaine from exogenously provided choline. J. Bacteriol. 176, 5364-5371. doi: 10.1128/jb.176.17.5364-5371. 1994

Bolen, D. W., and Baskakov, I. V. (2001). The osmophobic effect: natural selection of a thermodynamic force in protein folding. J. Mol. Biol. 310, 955-963. doi: 10.1006/jmbi.2001.4819

Boos, W., Ehmann, U., Forkl, H., Klein, W., Rimmele, M., and Postma, P. (1990). Trehalose transport and metabolism in Escherichia coli. J. Bacteriol. 172, 34503461. doi: 10.1128/jb.172.6.3450-3461.1990

\section{AUTHOR CONTRIBUTIONS}

AV and EB conceived and supervised this study. ML, TH, and CS-P conducted the experiments and analyzed their results. JH helped with the interpretation of genomic data. $\mathrm{TH}, \mathrm{JH}, \mathrm{AV}$, and EB wrote the paper.

\section{FUNDING}

Work conducted at the University of Seville (Spain), was supported by grants from the Spanish Ministry of Economy and Competitiveness (CGL2013-46941-P and CGL2017-83385P), FEDER funds and the Plan Andaluz de Investigación also supported this project (to AV). ML was recipient of a fellowship from the Junta de Andalucía. The work conducted at the University of Marburg (Germany) was financially supported by the Deutsche Forschungsgemeinschaft (DFG) through the Collaborative Research Consortium SFB 987 (to EB and JH).

\section{ACKNOWLEDGMENTS}

We greatly appreciate the kind help of Vickie Koogle in the language editing of our manuscript. We thank bitop AG (Witten, Germany) for generously providing us with samples of ectoine and hydroxyectoine.

\section{SUPPLEMENTARY MATERIAL}

The Supplementary Material for this article can be found online at: https://www.frontiersin.org/articles/10.3389/fmicb. 2018.00108/full\#supplementary-material

Booth, I. R. (2014). Bacterial mechanosensitive channels: progress towards an understanding of their roles in cell physiology. Curr. Opin. Microbiol. 18, 16-22. doi: 10.1016/j.mib.2014.01.005

Bourne, Y., and Henrissat, B. (2001). Glycoside hydrolases and glycosyltransferases: families and functional modules. Curr. Opin. Struct. Biol. 11, 593-600. doi: 10.1016/S0959-440X(00)00253-0

Bremer, E., and Krämer, R. (2000). "Coping with osmotic challenges: osmoregulation through accumulation and release of compatible solutes," in Bacterial Stress Responses, eds G. Storz and R. Hengge-Aronis (Washington, DC: ASM Press), 79-97.

Brill, J., Hoffmann, T., Bleisteiner, M., and Bremer, E. (2011). Osmotically controlled synthesis of the compatible solute proline is critical for cellular defense of Bacillus subtilis against high osmolarity. J. Bacteriol. 193, 5335-5346. doi: 10.1128/JB.05490-11

Broy, S., Chen, C., Hoffmann, T., Brock, N. L., Nau-Wagner, G., Jebbar, M., et al. (2015). Abiotic stress protection by ecologically abundant dimethylsulfoniopropionate and its natural and synthetic derivatives: insights from Bacillus subtilis. Environ. Microbiol. 7, 2362-2378. doi: 10.1111/1462-2920.12698

Bursy, J., Pierik, A. J., Pica, N., and Bremer, E. (2007). Osmotically induced synthesis of the compatible solute hydroxyectoine is mediated by an evolutionarily conserved ectoine hydroxylase. J. Biol. Chem. 282, 31147-31155. doi: 10.1074/jbc.M704023200

Calamita, G. (2000). The Escherichia coli aquaporin-Z water channel. Mol. Microbiol. 37, 254-262. doi: 10.1046/j.1365-2958.2000.02016.x 
Canovas, D., Vargas, C., Iglesias-Guerra, F., Csonka, L. N., Rhodes, D., Ventosa, A., et al. (1997). Isolation and characterization of salt-sensitive mutants of the moderate halophile Halomonas elongata and cloning of the ectoine synthesis genes. J. Biol. Chem. 272, 25794-25801. doi: 10.1074/jbc.272.41.25794

Carini, P., Campbell, E. O., Morre, J., Sanudo-Wilhelmy, S. A., Thrash, J. C., Bennett, S. E., et al. (2014). Discovery of a SAR11 growth requirement for thiamin's pyrimidine precursor and its distribution in the Sargasso Sea. ISME J. 8, 1727-1738. doi: 10.1038/ismej.2014.61

Csonka, L. N. (1989). Physiological and genetic responses of bacteria to osmotic stress. Microbiol. Rev. 53, 121-147.

Czech, L., Poehl, S., Hub, P., Stoeveken, N., and Bremer, E. (2018). Tinkering with osmotically controlled transcription allows enhanced production and excretion of ectoine and hydroxyectoine from a microbial cell factory. Appl. Environ. Microbiol. 84:e01772-17. doi: 10.1128/AEM.01772-17

da Costa, M. S., Santos, H., and Galinski, E. A. (1998). An overview of the role and diversity of compatible solutes in Bacteria and Archaea. Adv. Biochem. Eng. Biotechnol. 61, 117-153. doi: 10.1007/BFb0102291

Delano, W. L. (2002). The PyMol Molecular Graphics System. San Carlos, CA: Delano Scientific.

Deole, R., Challacombe, J., Raiford, D. W., and Hoff, W. D. (2013). An extremely halophilic proteobacterium combines a highly acidic proteome with a low cytoplasmic potassium content. J. Biol. Chem. 288, 581-588. doi: 10.1074/jbc. M112.420505

Dinnbier, U., Limpinsel, E., Schmid, R., and Bakker, E. P. (1988). Transient accumulation of potassium glutamate and its replacement by trehalose during adaptation of growing cells of Escherichia coli K-12 to elevated sodium chloride concentrations. Arch. Microbiol. 150, 348-357. doi: 10.1007/BF0040 8306

Diskowski, M., Mikusevic, V., Stock, C., and Hänelt, I. (2015). Functional diversity of the superfamily of $\mathrm{K}^{+}$transporters to meet various requirements. Biol. Chem. 396, 1003-1014. doi: 10.1515/hsz-2015-0123

Epstein, W. (2003). The roles and regulation of potassium in bacteria. Prog. Nucleic Acid Res. Mol. Biol. 75, 293-320. doi: 10.1016/S0079-6603(03)75008-9

Fernandez, A. B., Ghai, R., Martin-Cuadrado, A. B., Sanchez-Porro, C., Rodriguez-Valera, F., and Ventosa, A. (2014a). Prokaryotic taxonomic and metabolic diversity of an intermediate salinity hypersaline habitat assessed by metagenomics. Front. Microbiol. 5:196. doi: 10.3389/fmicb.2014.00196

Fernandez, A. B., Vera-Gargallo, B., Sanchez-Porro, C., Ghai, R., Papke, R. T., Rodriguez-Valera, F., et al. (2014b). Comparison of prokaryotic community structure from Mediterranean and Atlantic saltern concentrator ponds by a metagenomic approach. Front. Microbiol. 5:196. doi: 10.3389/fmicb.2014.00196

Fujisawa, M., Ito, M., and Krulwich, T. A. (2007). Three two-component transporters with channel-like properties have monovalent cation/proton antiport activity. Proc. Natl. Acad. Sci. U.S.A. 104, 13289-13294. doi: 10.1073/ pnas.0703709104

Galinski, E. A., and Trüper, H. G. (1994). Microbial behaviour in salt-stressed ecosystems. FEMS Microbiol. Rev. 15, 95-108. doi: 10.1111/j.1574-6976.1994. tb00128.x

Garcia-Estepa, R., Argandona, M., Reina-Bueno, M., Capote, N., IglesiasGuerra, F., Nieto, J. J., et al. (2006). The ectD gene, which is involved in the synthesis of the compatible solute hydroxyectoine, is essential for thermoprotection of the halophilic bacterium Chromohalobacter salexigens. J. Bacteriol. 188, 3774-3784. doi: 10.1128/JB.00136-06

Ghai, R., Pasic, L., Fernandez, A. B., Martin-Cuadrado, A. B., Mizuno, C. M., McMahon, K. D., et al. (2011). New abundant microbial groups in aquatic hypersaline environments. Sci. Rep. 1:135. doi: 10.1038/srep00135

Giaever, H. M., Styrvold, O. B., Kaasen, I., and Strom, A. R. (1988). Biochemical and genetic characterization of osmoregulatory trehalose synthesis in Escherichia coli. J. Bacteriol. 170, 2841-2849. doi: 10.1128/jb.170.6.2841-2849.1988

Gorecki, K., Hagerhall, C., and Drakenberg, T. (2014). The $\mathrm{Na}^{+}$transport in grampositive bacteria defect in the Mrp antiporter complex measured with $23 \mathrm{Na}$ nuclear magnetic resonance. Anal. Biochem. 445, 80-86. doi: 10.1016/j.ab.2013. 10.003

Grammann, K., Volke, A., and Kunte, H. J. (2002). New type of osmoregulated solute transporter identified in halophilic members of the bacteria domain: TRAP transporter TeaABC mediates uptake of ectoine and hydroxyectoine in Halomonas elongata DSM 2581(T). J. Bacteriol. 184, 3078-3085. doi: 10.1128/ JB.184.11.3078-3085.2002
Grant, W. D. (2004). Life at low water activity. Philos. Trans. R. Soc. Lond. B Biol. Sci. 359, 1249-1267. doi: 10.1098/rstb.2004.1502

Grote, J., Thrash, J. C., Huggett, M. J., Landry, Z. C., Carini, P., Giovannoni, S. J., et al. (2012). Streamlining and core genome conservation among highly divergent members of the SAR11 clade. mBio 3:e0252-12. doi: 10.1128/mBio. 00252-12

Haardt, M., Kempf, B., Faatz, E., and Bremer, E. (1995). The osmoprotectant proline betaine is a major substrate for the binding-protein-dependent transport system ProU of Escherichia coli K-12. Mol. Gen. Genet. 246, 783-786. doi: 10.1007/BF00290728

Hedfalk, K., Tornroth-Horsefield, S., Nyblom, M., Johanson, U., Kjellbom, P., and Neutze, R. (2006). Aquaporin gating. Curr. Opin. Struct. Biol. 16, 447-456. doi: 10.1016/j.sbi.2006.06.009

Hengge-Aronis, R. (2002). Signal transduction and regulatory mechanisms involved in control of the sigma(S) (RpoS) subunit of RNA polymerase. Microbiol. Mol. Biol. Rev. 66, 373-395. doi: 10.1128/MMBR.66.3.373-395. 2002

Hengge-Aronis, R., Klein, W., Lange, R., Rimmele, M., and Boos, W. (1991). Trehalose synthesis genes are controlled by the putative sigma factor encoded by rpoS and are involved in stationary-phase thermotolerance in Escherichia coli. J. Bacteriol. 173, 7918-7924. doi: 10.1128/jb.173.24.7918-7924.1991

Hoffmann, T., and Bremer, E. (2011). Protection of Bacillus subtilis against cold stress via compatible-solute acquisition. J. Bacteriol. 193, 1552-1562. doi: 10. 1128/JB.01319-10

Hoffmann, T., and Bremer, E. (2016). "Management of osmotic stress by Bacillus subtilis: genetics and physiology," in Stress and Environmental Regulation of Gene Expression and Adaptation in Bacteria, ed. F. J. de Bruijn (Hoboken, NJ: Wiley-Blackwell Publishers), 657-676. doi: 10.1002/9781119004813.ch63

Hoffmann, T., and Bremer, E. (2017). Guardians in a stressful world: the Opu family of compatible solute transporters from Bacillus subtilis. Biol. Chem. 398, 193-214. doi: 10.1515/hsz-2016-0265

Hoffmann, T., Warmbold, B., Smits, S. H. J., Tschapek, B., Ronzheimer, S., Bashir, A., et al. (2017). Arsenobetaine: an ecophysiologically important organoarsenical confers cytoprotection against osmotic stress and growth temperature extremes. Environ. Microbiol. 20, 305-323. doi: 10.1111/14622920.13999

Hoffmann, T., Wensing, A., Brosius, M., Steil, L., Völker, U., and Bremer, E. (2013). Osmotic control of opuA expression in Bacillus subtilis and its modulation in response to intracellular glycine betaine and proline pools. J. Bacteriol. 195, 510-522. doi: 10.1128/JB.01505-12

Holtmann, G., Bakker, E. P., Uozumi, N., and Bremer, E. (2003). KtrAB and KtrCD: two $\mathrm{K}^{+}$uptake systems in Bacillus subtilis and their role in adaptation to hypertonicity. J. Bacteriol. 185, 1289-1298. doi: 10.1128/JB.185.4.1289-1298. 2003

Höppner, A., Widderich, N., Lenders, M., Bremer, E., and Smits, S. H. J. (2014). Crystal structure of the ectoine hydroxylase, a snapshot of the active site. J. Biol. Chem. 289, 29570-29583. doi: 10.1074/Jbc.M114.576769

Horn, C., Sohn-Bösser, L., Breed, J., Welte, W., Schmitt, L., and Bremer, E. (2006). Molecular determinants for substrate specificity of the ligand-binding protein OpuAC from Bacillus subtilis for the compatible solutes glycine betaine and proline betaine. J. Mol. Biol. 357, 592-606. doi: 10.1016/j.jmb.2005. 12.085

Ignatova, Z., and Gierasch, L. M. (2006). Inhibition of protein aggregation in vitro and in vivo by a natural osmoprotectant. Proc. Natl. Acad. Sci. U.S.A. 103, 13357-13361. doi: 10.1073/pnas.0603772103

Ito, M., Morino, M., and Krulwich, T. A. (2017). Mrp antiporters have important roles in diverse bacteria and archaea. Front. Microbiol. 8:2325. doi: 10.3389/ fmicb.2017.02325

Jebbar, M., Sohn-Bösser, L., Bremer, E., Bernard, T., and Blanco, C. (2005). Ectoineinduced proteins in Sinorhizobium meliloti include an ectoine ABC-type transporter involved in osmoprotection and ectoine catabolism. J. Bacteriol. 187, 1293-1304. doi: 10.1128/JB.187.4.1293-1304.2005

Kanehisa, M., Goto, S., Sato, Y., Furumichi, M., and Tanabe, M. (2012). KEGG for integration and interpretation of large-scale molecular data sets. Nucleic Acids Res. 40, D109-D114. doi: 10.1093/nar/gkr988

Kappes, R. M., Kempf, B., and Bremer, E. (1996). Three transport systems for the osmoprotectant glycine betaine operate in Bacillus subtilis: characterization of OpuD. J. Bacteriol. 178, 5071-5079. doi: 10.1128/jb.178.17.5071-5079.1996 
Kempf, B., and Bremer, E. (1995). OpuA, an osmotically regulated binding proteindependent transport system for the osmoprotectant glycine betaine in Bacillus subtilis. J. Biol. Chem. 270, 16701-16713. doi: 10.1074/jbc.270.28.16701

Kempf, B., and Bremer, E. (1998). Uptake and synthesis of compatible solutes as microbial stress responses to high osmolality environments. Arch. Microbiol. 170, 319-330. doi: 10.1007/s002030050649

Klähn, S., and Hagemann, M. (2011). Compatible solute biosynthesis in cyanobacteria. Environ. Microbiol. 13, 551-562. doi: 10.1111/j.1462-2920.2010. 02366.x

Krämer, R. (2010). Bacterial stimulus perception and signal transduction: response to osmotic stress. Chem. Rec. 10, 217-229. doi: 10.1002/tcr.201000005

Kuhlmann, A. U., and Bremer, E. (2002). Osmotically regulated synthesis of the compatible solute ectoine in Bacillus pasteurii and related Bacillus spp. Appl. Environ. Microbiol. 68, 772-783. doi: 10.1128/AEM.68.2.772-783. 2002

Kuhlmann, A. U., Bursy, J., Gimpel, S., Hoffmann, T., and Bremer, E. (2008). Synthesis of the compatible solute ectoine in Virgibacillus pantothenticus is triggered by high salinity and low growth temperature. Appl. Environ. Microbiol. 74, 4560-4563. doi: 10.1128/AEM.00492-08

Kuhlmann, A. U., Hoffmann, T., Bursy, J., Jebbar, M., and Bremer, E. (2011). Ectoine and hydroxyectoine as protectants against osmotic and cold stress: uptake through the SigB-controlled betaine-choline-carnitine transporter-type carrier EctT from Virgibacillus pantothenticus. J. Bacteriol. 193, 4699-4708. doi: 10.1128/JB.05270- 11

Kuhlmann, S. I., Terwisscha van Scheltinga, A. C., Bienert, R., Kunte, H. J., and Ziegler, C. (2008). 1.55 A structure of the ectoine binding protein TeaA of the osmoregulated TRAP-transporter TeaABC from Halomonas elongata. Biochemistry 47, 9475-9485. doi: 10.1021/bi8006719

Lecher, J., Pittelkow, M., Zobel, S., Bursy, J., Bonig, T., Smits, S. H., et al. (2009). The crystal structure of UehA in complex with ectoine-A comparison with other TRAP-T binding proteins. J. Mol. Biol. 389, 58-73. doi: 10.1016/j.jmb.2009. 03.077

León, M. J., Aldeguer-Riquelme, B., Anton, J., Sanchez-Porro, C., and Ventosa, A. (2017). Spiribacter aquaticus sp. nov., a novel member of the genus Spiribacter isolated from a saltern. Int. J. Syst. Evol. Microbiol. 67, 2947-2952. doi: 10.1099/ ijsem.0.002053

León, M. J., Fernandez, A. B., Ghai, R., Sanchez-Porro, C., Rodriguez-Valera, F., and Ventosa, A. (2014). From metagenomics to pure culture: isolation and characterization of the moderately halophilic bacterium Spiribacter salinus gen. nov., sp. nov. Appl. Environ. Microbiol. 80, 3850-3857. doi: 10.1128/AEM. 00430- 14

León, M. J., Ghai, R., Fernandez, A. B., Sanchez-Porro, C., Rodriguez-Valera, F., and Ventosa, A. (2013). Draft genome of Spiribacter salinus M19-40, an abundant gammaproteobacterium in aquatic hypersaline environments. Genome Announc. 1:e00179-12. doi: 10.1128/genomeA.00179-12

León, M. J., Rodriguez-Olmos, A., Sanchez-Porro, C., Lopez-Perez, M., RodriguezValera, F., Soliveri, J., et al. (2015). Spiribacter curvatus sp. nov., a moderately halophilic bacterium isolated from a saltern. Int. J. Syst. Evol. Microbiol. 65, 4638-4643. doi: 10.1099/ijsem.0.000621

León, M. J., Vera-Gargallo, B., Sanchez-Porro, C., and Ventosa, A. (2016). Spiribacter roseus sp. nov., a moderately halophilic species of the genus Spiribacter from salterns. Int. J. Syst. Evol. Microbiol. 66, 4218-4224. doi: 10. 1099/ijsem.0.001338

Lopez-Perez, M., Ghai, R., León, M. J., Rodriguez-Olmos, A., Copa-Patino, J. L., Soliveri, J., et al. (2013). Genomes of "Spiribacter", a streamlined, successful halophilic bacterium. BMC Genomics 14:787. doi: 10.1186/1471-216414-787

Louis, P., and Galinski, E. A. (1997). Characterization of genes for the biosynthesis of the compatible solute ectoine from Marinococcus halophilus and osmoregulated expression in Escherichia coli. Microbiology 143, 1141-1149. doi: 10.1099/00221287-143-4-1141

Lucht, J. M., and Bremer, E. (1994). Adaptation of Escherichia coli to high osmolarity environments: osmoregulation of the high-affinity glycine betaine transport system ProU. FEMS Microbiol. Rev. 14, 3-20. doi: 10.1111/j.15746976.1994.tb00067.x

Lundberg, M. E., Becker, E. C., and Choe, S. (2013). MstX and a putative potassium channel facilitate biofilm formation in Bacillus subtilis. PLOS ONE 8:e60993. doi: 10.1371/journal.pone.0060993
Morbach, S., and Krämer, R. (2002). Body shaping under water stress: osmosensing and osmoregulation of solute transport in bacteria. Chembiochem 3, 384-397. doi: 10.1002/1439-7633(20020503)3:5<384::AID-CBIC384>3.0.CO;2-H

Mulligan, C., Fischer, M., and Thomas, G. H. (2011). Tripartite ATP-independent periplasmic (TRAP) transporters in bacteria and archaea. FEMS Microbiol. Rev. 35, 68-86. doi: 10.1111/j.1574-6976.2010.00236.x

Nau-Wagner, G., Boch, J., Le Good, J. A., and Bremer, E. (1999). High-affinity transport of choline-O-sulfate and its use as a compatible solute in Bacillus subtilis. Appl. Environ. Microbiol. 65, 560-568.

Nordberg, H., Cantor, M., Dusheyko, S., Hua, S., Poliakov, A., Shabalov, I., et al. (2013). The genome portal of the Department of Energy Joint Genome Institute: 2014 updates. Nucleic Acids Res. 42, D26-D31. doi: 10.1093/nar/gkt1069

Ochrombel, I., Becker, M., Krämer, R., and Marin, K. (2011). Osmotic stress response in C. glutamicum: impact of channel- and transporter-mediated potassium accumulation. Arch. Microbiol. 193, 787-796. doi: 10.1007/s00203011-0717-6

Ono, H., Sawada, K., Khunajakr, N., Tao, T., Yamamoto, M., Hiramoto, M., et al. (1999). Characterization of biosynthetic enzymes for ectoine as a compatible solute in a moderately halophilic eubacterium, Halomonas elongata. J. Bacteriol. 181, 91-99.

Oren, A. (2011). Thermodynamic limits to microbial life at high salt concentrations. Environ. Microbiol. 13, 1908-1923. doi: 10.1111/j.1462-2920. 2010.02365.x

Oren, A. (2013). Life at high salt concentrations, intracellular $\mathrm{KCl}$ concentrations, and acidic proteomes. Front. Microbiol. 4:315. doi: 10.3389/fmicb.2013. 00315

Pastor, J. M., Salvador, M., Argandona, M., Bernal, V., Reina-Bueno, M., Csonka, L. N., et al. (2010). Ectoines in cell stress protection: uses and biotechnological production. Biotechnol. Adv. 28, 782-801. doi: 10.1016/j.biotechadv.2010. 06.005

Poolman, B., Spitzer, J. J., and Wood, J. M. (2004). Bacterial osmosensing: roles of membrane structure and electrostatics in lipid-protein and protein-protein interactions. Biochim. Biophys. Acta 1666, 88-104. doi: 10.1016/j.bbamem.2004. 06.013

Popowich, A., Zhang, Q., and Le, X. C. (2016). Arsenobetaine: the ongoing mystery. Nat. Sci. Rev. 3, 451-458. doi: 10.1093/nsr/nww061

Prabhu, J., Schauwecker, F., Grammel, N., Keller, U., and Bernhard, M. (2004). Functional expression of the ectoine hydroxylase gene $(t h p D)$ from Streptomyces chrysomallus in Halomonas elongata. Appl. Environ. Microbiol. 70, 3130-3132. doi: 10.1128/AEM.70.5.3130-3132.2004

Pundir, S., Martin, M. J., and O'Donovan, C. (2017). UniProt protein knowledgebase. Methods Mol. Biol. 1558, 41-55. doi: 10.1007/978-1-49396783-4_2

Reshetnikov, A. S., Khmelenina, V. N., and Trotsenko, Y. A. (2006). Characterization of the ectoine biosynthesis genes of haloalkalotolerant obligate methanotroph "Methylomicrobium alcaliphilum 20Z". Arch. Microbiol. 184, 286-297. doi: 10.1007/s00203-005-0042-z

Reuter, K., Pittelkow, M., Bursy, J., Heine, A., Craan, T., and Bremer, E. (2010). Synthesis of 5-hydroxyectoine from ectoine: crystal structure of the non-heme iron(II) and 2-oxoglutarate-dependent dioxygenase EctD. PLOS ONE 5:e10647. doi: 10.1371/journal.pone.0010647

Roesser, M., and Müller, V. (2001). Osmoadaptation in bacteria and archaea: common principles and differences. Environ. Microbiol. 3, 743-754. doi: 10. 1046/j.1462-2920.2001.00252.x

Saum, S. H., Pfeiffer, F., Palm, P., Rampp, M., Schuster, S. C., Müller, V., et al. (2013). Chloride and organic osmolytes: a hybrid strategy to cope with elevated salinities by the moderately halophilic, chloride-dependent bacterium Halobacillus halophilus. Environ. Microbiol. 15, 1619-1633. doi: 10.1111/j.14622920.2012.02770.x

Schiefner, A., Breed, J., Bosser, L., Kneip, S., Gade, J., Holtmann, G., et al. (2004). Cation-pi interactions as determinants for binding of the compatible solutes glycine betaine and proline betaine by the periplasmic ligand-binding protein ProX from Escherichia coli. J. Biol. Chem. 279, 5588-5596. doi: 10.1074/jbc. M309771200

Schulz, A., Herrman, L., Freibert, S.-A., Bönig, T., Hoffmann, T., Riclea, R., et al. (2017a). Transcriptional regulation of ectoine catabolism in response to multiple metabolic and environmental cues. Environ. Microbiol. 19, 4599-4619. doi: $10.1111 / 1462-2920.13924$ 
Schulz, A., Stöveken, N., Binzen, I. M., Hoffmann, T., Heider, J., and Bremer, E. (2017b). Feeding on compatible solutes: a substrate-induced pathway for uptake and catabolism of ectoines and its genetic control by EnuR. Environ. Microbiol. 19, 926-946. doi: 10.1111/1462-2920.13414

Schwibbert, K., Marin-Sanguino, A., Bagyan, I., Heidrich, G., Lentzen, G., Seitz, H., et al. (2011). A blueprint of ectoine metabolism from the genome of the industrial producer Halomonas elongata DSM 2581 T. Environ. Microbiol. 13, 1973-1994. doi: 10.1111/j.1462-2920.2010.02336.x

Smits, S. H., Höing, M., Lecher, J., Jebbar, M., Schmitt, L., and Bremer, E. (2008). The compatible-solute-binding protein OpuAC from Bacillus subtilis: ligand binding, site-directed mutagenesis, and crystallographic studies. J. Bacteriol. 190, 5663-5671. doi: 10.1128/JB.00346-08

Stadmiller, S. S., Gorensek-Benitez, A. H., Guseman, A. J., and Pielak, G. J. (2017). Osmotic shock induced protein destabilization in living cells and its reversal by glycine betaine. J. Mol. Biol. 429, 1155-1161. doi: 10.1016/j.jmb.2017.03.001

Stöveken, N., Pittelkow, M., Sinner, T., Jensen, R. A., Heider, J., and Bremer, E. (2011). A specialized aspartokinase enhances the biosynthesis of the osmoprotectants ectoine and hydroxyectoine in Pseudomonas stutzeri A1501. J. Bacteriol. 193, 4456-4468. doi: 10.1128/JB.00345-11

Street, T. O., Bolen, D. W., and Rose, G. D. (2006). A molecular mechanism for osmolyte-induced protein stability. Proc. Natl. Acad. Sci. U.S.A. 103, 1399714002. doi: 10.1073/pnas.0606236103

Strom, A. R., and Kaasen, I. (1993). Trehalose metabolism in Escherichia coli: stress protection and stress regulation of gene expression. Mol. Microbiol. 8, 205-210. doi: 10.1111/j.1365-2958.1993.tb01564.x

Suh, S. S., Park, M., Hwang, J., Kil, E. J., Jung, S. W., Lee, S., et al. (2015). Seasonal dynamics of marine microbial community in the South Sea of Korea. PLOS ONE 10:e0131633. doi: 10.1371/journal.pone.0131633

Swan, B. K., Tupper, B., Sczyrba, A., Lauro, F. M., Martinez-Garcia, M., Gonzalez, J. M., et al. (2013). Prevalent genome streamlining and latitudinal divergence of planktonic bacteria in the surface ocean. Proc. Natl. Acad. Sci. U.S.A. 110, 11463-11468. doi: 10.1073/pnas. 1304246110

Swartz, T. H., Ikewada, S., Ishikawa, O., Ito, M., and Krulwich, T. A. (2005). The Mrp system: a giant among monovalent cation/proton antiporters? Extremophiles 9, 345-354. doi: 10.1007/s00792-005-0451-6

Szollosi, A., Vieira-Pires, R. S., Teixeira-Duarte, C. M., Rocha, R., and MoraisCabral, J. H. (2016). Dissecting the molecular mechanism of nucleotidedependent activation of the KtrAB $\mathrm{K}^{+}$transporter. PLOS Biol. 14:e1002356. doi: 10.1371/journal.pbio.1002356

Talon, R., Coquelle, N., Madern, D., and Girard, E. (2014). An experimental point of view on hydration/solvation in halophilic proteins. Front. Microbiol. 5:66. doi: $10.3389 /$ fmicb. 2014.00066

Tanghe, A., Van Dijck, P., and Thevelein, J. M. (2006). Why do microorganisms have aquaporins? Trends Microbiol. 14, 78-85. doi: 10.1016/j.tim.2005.12.001

Vargas, C., Argandona, M., Reina-Bueno, M., Rodriguez-Moya, J., FernandezAunion, C., and Nieto, J. J. (2008). Unravelling the adaptation responses to osmotic and temperature stress in Chromohalobacter salexigens, a bacterium with broad salinity tolerance. Saline Systems 4:14. doi: 10.1186/1746-1448-4-14

Vargas, C., Jebbar, M., Carrasco, R., Blanco, C., Calderon, M. I., Iglesias-Guerra, F., et al. (2006). Ectoines as compatible solutes and carbon and energy sources for the halophilic bacterium Chromohalobacter salexigens. J. Appl. Microbiol. 100, 98-107. doi: 10.1111/j.1365-2672.2005.02757.x

Ventosa, A., de la Haba, R. R., Sanchez-Porro, C., and Papke, R. T. (2015). Microbial diversity of hypersaline environments: a metagenomic approach. Curr. Opin. Microbiol. 25, 80-87. doi: 10.1016/j.mib.2015.05.002

Ventosa, A., Fernandez, A. B., León, M. J., Sanchez-Porro, C., and RodriguezValera, F. (2014). The Santa Pola saltern as a model for studying the microbiota of hypersaline environments. Extremophiles 18, 811-824. doi: 10.1007/s00792014-0681-6
Ventosa, A., Nieto, J. J., and Oren, A. (1998). Biology of moderately halophilic aerobic bacteria. Microbiol. Mol. Biol. Rev. 62, 504-544.

Warden, A. C., Williams, M., Peat, T. S., Seabrook, S. A., Newman, J., Dojchinov, G., et al. (2015). Rational engineering of a mesohalophilic carbonic anhydrase to an extreme halotolerant biocatalyst. Nat. Commun. 6:10278. doi: 10.1038/ ncomms 10278

Welsh, D. T. (2000). Ecological significance of compatible solute accumulation by micro-organisms: from single cells to global climate. FEMS Microbiol. Rev. 24, 263-290. doi: 10.1111/j.1574-6976.2000.tb00542.x

Whatmore, A. M., Chudek, J. A., and Reed, R. H. (1990). The effects of osmotic upshock on the intracellular solute pools of Bacillus subtilis. J. Gen. Microbiol. 136, 2527-2535. doi: 10.1099/00221287-136-12-2527

Widderich, N., Czech, L., Elling, F. J., Könneke, M., Stöveken, N., Pittelkow, M., et al. (2016). Strangers in the archaeal world: osmostress-responsive biosynthesis of ectoine and hydroxyectoine by the marine thaumarchaeon Nitrosopumilus maritimus. Environ. Microbiol. 18, 1227-1248. doi: 10.1111/ 1462-2920.13156

Widderich, N., Höppner, A., Pittelkow, M., Heider, J., Smits, S. H., and Bremer, E. (2014). Biochemical properties of ectoine hydroxylases from extremophiles and their wider taxonomic distribution among microorganisms. PLOS ONE 9:e93809. doi: 10.1371/journal.pone.0093809

Wolters, J. C., Berntsson, R. P., Gul, N., Karasawa, A., Thunnissen, A. M., Slotboom, D. J., et al. (2010). Ligand binding and crystal structures of the substrate-binding domain of the ABC transporter OpuA. PLOS ONE 5:e10361. doi: 10.1371/journal.pone.0010361

Wood, J. M. (2011). Bacterial osmoregulation: a paradigm for the study of cellular homeostasis. Annu. Rev. Microbiol. 65, 215-238. doi: 10.1146/annurev-micro090110-102815

Wood, J. M., Bremer, E., Csonka, L. N., Krämer, R., Poolman, B., van der Heide, T., et al. (2001). Osmosensing and osmoregulatory compatible solute accumulation by bacteria. Comp. Biochem. Physiol. A Mol. Integr. Physiol. 130, 437-460. doi: 10.1016/S1095-6433(01)00442-1

Yancey, P. H. (2005). Organic osmolytes as compatible, metabolic and counteracting cytoprotectants in high osmolarity and other stresses. J. Exp. Biol. 208, 2819-2830. doi: 10.1242/jeb.01730

Youssef, N. H., Savage-Ashlock, K. N., McCully, A. L., Luedtke, B., Shaw, E. I., Hoff, W. D., et al. (2014). Trehalose/2-sulfotrehalose biosynthesis and glycine-betaine uptake are widely spread mechanisms for osmoadaptation in the Halobacteriales. ISME J. 8, 636-649. doi: 10.1038/ismej. 2013.165

Zaccai, G., Bagyan, I., Combet, J., Cuello, G. J., Deme, B., Fichou, Y., et al. (2016). Neutrons describe ectoine effects on water H-bonding and hydration around a soluble protein and a cell membrane. Sci. Rep. 6:31434. doi: 10.1038/srep 31434

Ziegler, C., Bremer, E., and Krämer, R. (2010). The BCCT family of carriers: from physiology to crystal structure. Mol. Microbiol. 78, 13-34. doi: 10.1111/j.13652958.2010.07332.x

Conflict of Interest Statement: The authors declare that the research was conducted in the absence of any commercial or financial relationships that could be construed as a potential conflict of interest.

Copyright (c) 2018 León, Hoffmann, Sánchez-Porro, Heider, Ventosa and Bremer. This is an open-access article distributed under the terms of the Creative Commons Attribution License (CC BY). The use, distribution or reproduction in other forums is permitted, provided the original author(s) and the copyright owner are credited and that the original publication in this journal is cited, in accordance with accepted academic practice. No use, distribution or reproduction is permitted which does not comply with these terms. 